\title{
On Griess Algebras
}

Michael ROITMAN

Department of Mathematics, Kansas State University, Manhattan, KS 66506 USA

E-mail: misha.roitman@gmail.com

Received February 29, 2008, in final form July 28, 2008; Published online August 13, 2008

Original article is available at http://www.emis.de/journals/SIGMA/2008/057/

\begin{abstract}
In this paper we prove that for any commutative (but in general non-associative) algebra $A$ with an invariant symmetric non-degenerate bilinear form there is a graded vertex algebra $V=V_{0} \oplus V_{2} \oplus V_{3} \oplus \cdots$, such that $\operatorname{dim} V_{0}=1$ and $V_{2}$ contains $A$. We can choose $V$ so that if $A$ has a unit $e$, then $2 e$ is the Virasoro element of $V$, and if $G$ is a finite group of automorphisms of $A$, then $G$ acts on $V$ as well. In addition, the algebra $V$ can be chosen with a non-degenerate invariant bilinear form, in which case it is simple.
\end{abstract}

Key words: vertex algebra; Griess algebra

2000 Mathematics Subject Classification: 17B69

\section{Introduction}

A vertex algebra $V$ is a linear space, endowed with infinitely many bilinear products $(n)$ : $V \otimes V \rightarrow V$ and a unit $\mathbb{1} \in V$, satisfying certain axioms, see Section 2.1. In this paper we deal with graded vertex algebras $V=\bigoplus_{i \in \mathbb{Z}} V_{i}$, so that $V_{i}(n) V_{j} \subseteq V_{i+j-n-1}$ and $\mathbb{1} \in V_{0}$. A vertex algebra is called OZ (abbreviation of "One-Zero") [12] if it is graded so that $\operatorname{dim} V_{0}=1$ and $V_{i}=0$ for $i=1$ or $i<0$. If $V$ is an OZ vertex algebra, then [9] $V_{2}$ is a commutative (but not necessary associative) algebra with respect to the product (1) $: V_{2} \otimes V_{2} \rightarrow V_{2}$, with an invariant symmetric bilinear form (i.e. such that $\langle a b \mid c\rangle=\langle a \mid b c\rangle$ ), given by the product (3) : $V_{2} \otimes V_{2} \rightarrow V_{0}$. It is called the Griess algebra of $V .^{1}$

\subsection{Formulation of the results}

In this paper we prove the following result.

\section{Theorem 1.1.}

a. For any commutative algebra $A$ with a symmetric invariant non-degenerate bilinear form there is a simple $O Z$ vertex algebra $V$ such that $A \subseteq V_{2}$.

b. If $A$ has a unit e, then $V$ can be chosen so that $\omega=2 e$ is a Virasoro element of $V$ (see Section 2.1 for the definition).

c. If $G \subset$ Aut $A$ is a finite group of automorphisms of $A$, then $V$ can be chosen so that $G \subset$ Aut $V$.

We prove this theorem under the assumption, that the ground field $\mathbb{k}$ is a subfield of $\mathbb{C}$, since our proof uses some analytic methods (see Section 3). However, we believe that the statement can be generalized to an arbitrary field of characteristic 0 . Also, the assumption that the form is non-degenerate does not seem to be very essential.

\footnotetext{
${ }^{\star}$ This paper is a contribution to the Special Issue on Kac-Moody Algebras and Applications. The full collection is available at http://www.emis.de/journals/SIGMA/Kac-Moody_algebras.html

${ }^{1}$ We note that the term "Griess algebra" might not be the most successful one, as the original Griess algebra [11] is not quite a Griess algebra in our sense.
} 
In fact we suggest that the following conjecture might be true:

\section{Conjecture 1.1.}

a. For any commutative algebra $A$ with a symmetric invariant bilinear form there is an $O Z$ vertex algebra $V$ such that $A=V_{2}$.

b. If $\operatorname{dim} A<\infty$, then $V$ can be chosen so that $\operatorname{dim} V_{n}<\infty$ for $n=3,4,5, \ldots$.

It follows from Theorem 1.1 that there are no Griess identities other than commutativity, in other words, for any non-trivial identity in the variety of commutative algebras with symmetric invariant bilinear forms there is a Griess algebra in which this identity does not hold.

Here we outline our construction of $V$. First we construct a vertex algebra $B=B_{0} \oplus B_{2} \oplus$ $B_{3} \oplus \cdots$, such that $B_{0}$ is a polynomial algebra and $A \subset B_{2}$. In fact we construct the vertex coalgebra of correlation functions on $B$, defined in Section 4 , and then derive $B$ from it. After that we find a suitable invariant bilinear form $\langle\cdot \mid \cdot\rangle$ on $B$ and set $V=B / \operatorname{Ker}\langle\cdot \mid \cdot\rangle$.

We remark that our methods would perfectly work for a more general problem: Given an "initial segment" $A_{0} \oplus A_{1} \oplus \cdots \oplus A_{m}$ of a vertex algebra, closed under those of the vertex operations $(n)$ that make sense, find a vertex algebra $V=\bigoplus_{d \geqslant 0} V_{d}$ such that $V_{d} \supset A_{d}$ for $0 \leqslant d \leqslant m$.

\subsection{Previously known results}

Probably the most famous example of OZ vertex algebras is the Moonshine module $V^{\natural}$, constructed by Frenkel, Lepowsky and Meurman in [8,9], see also [1,2]. Its Griess algebra $V_{2}^{\natural}$ has dimension 196884 , and differs from the original 196883 -dimensional commutative algebra constructed by Griess [11] by having an additional identity element. The automorphism group of $V^{\natural}$ and of $V_{2}^{\natural}$ is the Fischer-Griess Monster [9, 11, 25]. It is proved by Dong et al. [3, 6], that the representations of $V^{\natural}$ are completely reducible, and the only irreducible representation is $V^{\natural}$ itself. The present research was primarily motivated by this construction.

Another example of $\mathrm{OZ}$ vertex algebra is a Virasoro vertex algebra Vir [10, 26]. It is generated by a single Virasoro element $\omega \in \operatorname{Vir}_{2}$ so that the Griess algebra of Vir is $\mathbb{k} \omega$. The representation theory of the algebras Vir is investigated in [26].

If $A$ is associative, than it is well known that $A$ can appear as a Griess algebra, see [15, 27]. Lam [16] also showed the same for a simple Jordan algebra of type $A, B$ or $C$. Other interesting examples of $\mathrm{OZ}$ vertex algebras and their Griess algebras can be found in [12].

We remark that if a vertex algebra $V$ is graded so that $V_{n}=0$ for $n<0$ and $\operatorname{dim} V_{0}=1$, then $V_{1}$ is a Lie algebra with respect to the product (0), with invariant bilinear form given by product (1). The analogous problem of finding a vertex algebra $V$ such that $V_{1}$ is a given Lie algebra has a well-know solution: to every Lie algebra $L$ with an invariant bilinear form there corresponds an affine Lie algebra $\widehat{L}$, so that a certain highest weight $\widehat{L}$-module has the desired vertex algebra structure $[9,10]$.

\subsection{Organization of the manuscript}

In Section 2 we recall some basic definitions and notations of the theory of vertex algebras. Then in Section 3 we consider a class of rational functions that we call regular. The correlation functions of a sufficiently nice vertex algebra will belong to that class. Then in Section 3.4 we define a more narrow class of admissible functions. The correlation functions of the algebras $B$ and $V$ that we construct later are admissible. In Section 4 we introduce a notion of vertex coalgebra of correlation functions, and show how to reconstruct a vertex algebra by its coalgebra of correlation functions. In Section 4.6 we show that in some important cases the component of degree 0 of such vertex algebra is isomorphic to a polynomial algebra. In Section 5 we study some 
easy properties of $\mathrm{OZ}$ vertex algebras, in particular (in Section 5.2) investigate the behavior of the correlation functions in the presence of a Virasoro element. Then in Section 6 we construct certain vertex algebra $B$ using the coalgebra techniques developed in Section 4 and show (in Section 6.1) how the existence of the algebra $B$ implies Theorem 1.1.

\subsection{Further questions}

Though the methods used in this paper are very explicit, it seems that the OZ vertex algebras constructed here are of "generic type", i.e. they probably don't have these nice properties people are looking for in vertex algebra theory - for example, an interesting representation theory, various finiteness conditions, controllable Zhu algebra, etc. It would be extremely interesting to recover the OZ vertex algebras mentioned above using our approach, especially the Moonshine module $V^{\natural}$.

Also, it would be very interesting to see whether any properties of the commutative algebra $A$ (e.g. if $A$ is a Jordan algebra) imply any properties of the $\mathrm{OZ}$ vertex algebra $V$, constructed in Theorem 1.1.

\section{General facts about vertex algebras}

Here we fix the notations and give some minimal definitions. For more details on vertex algebras the reader can refer to the books $[9,14,17,27]$. Unless otherwise noted, we assume that all algebras and spaces are over a ground field $\mathbb{k} \subset \mathbb{C}$.

\subsection{Definition of vertex algebras}

Definition 2.1. A vertex algebra is a linear space $V$ equipped with a family of bilinear products $a \otimes b \mapsto a(n) b$, indexed by integer parameter $n$, and with an element $\mathbb{1} \in V$, called the unit, satisfying the identities (V1)-(V4) below. Let $D: V \rightarrow V$ be the map defined by $D a=a(-2) \mathbb{1}$. Then the identities are:

(V1) $a(n) b=0$ for $n \gg 0$,

(V2) $\mathbb{1}(n) a=\delta_{n,-1} a \quad$ and $\quad a(n) \mathbb{1}=\frac{1}{(-n-1) !} D^{-n-1} a$,

$$
\begin{aligned}
& D(a(n) b)=(D a)(n) b+a(n)(D b) \quad \text { and } \quad(D a)(n) b=-n a(n-1) b \\
& a(m)(b(n) c)-b(n)(a(m) c)=\sum_{s \geqslant 0}\left(\begin{array}{c}
m \\
s
\end{array}\right)(a(s) b)(m+n-s) c
\end{aligned}
$$

for all $a, b, c \in V$ and $m, n \in \mathbb{Z}$.

Another way of defining vertex algebras is by using the generating series

$$
Y: V \rightarrow \operatorname{Hom}(V, V((z)))
$$

defined for $a \in V$ by

$$
Y(a, z)=\sum_{n \in \mathbb{Z}} a(n) z^{-n-1}
$$

where $a(n): V \rightarrow V$ is the operator given by $b \mapsto a(n) b$, and $z$ is a formal variable. The most important property of these maps is that they are local: for any $a, b \in V$ there is $N \geqslant 0$ such that

$$
[Y(a, w), Y(b, z)](w-z)^{N}=0 .
$$


In fact, this is the only essential condition that one needs to postulate to define vertex algebras $[14,19]$. The minimal number $N$ for which $(2.1)$ holds is called the locality of $a$ and $b$, and is denoted by $\operatorname{loc}(a, b)$.

Remark 2.1. One could extend this definition to allow a negative locality (see [23]), so that

$$
\operatorname{loc}(a, b)=\min \{n \in \mathbb{Z} \mid a(m) b=0 \forall m \geqslant n\} .
$$

In terms of the series $Y$, the identities (V2) and (V3) read respectively

$$
Y(\mathbb{1}, z)=i d, \quad Y(a, z) \mathbb{1}=\exp (D z) a
$$

and

$$
Y(D a, z)=[D, Y(a, z)]=\partial_{z} Y(a, z) .
$$

Among other identities that hold in vertex algebras are the quasi-symmetry

$$
a(n) b=-\sum_{i \geqslant 0}(-1)^{n+i} D^{(i)}(b(n+i) a),
$$

and the associativity identity

$$
\begin{aligned}
(a(m) b)(n) c= & \sum_{s \geqslant 0}(-1)^{s}\left(\begin{array}{c}
m \\
s
\end{array}\right) a(m-s)(b(n+s) c) \\
& -\sum_{s \leqslant m}(-1)^{s}\left(\begin{array}{c}
m \\
m-s
\end{array}\right) b(n+s)(a(m-s) c) .
\end{aligned}
$$

For $m \geqslant 0$ this simplifies to

$$
(a(m) b)(n) c=\sum_{s=0}^{m}(-1)^{s}\left(\begin{array}{c}
m \\
s
\end{array}\right)[a(m-s), b(n+s)] c,
$$

which can also be derived from the identity (V4) of Definition 2.1 by some simple manipulations.

A vertex algebra $V$ is called graded (by the integers) if $V=\bigoplus_{i \in \mathbb{Z}} V_{i}$ is a graded space, so that $V_{i}(n) V_{j} \subseteq V_{i+j-n-1}$ and $\mathbb{1} \in V_{0}$. It is often assumed that a vertex algebra $V$ is graded and $V_{2}$ contains a special element $\omega$, called the Virasoro element of $V$, such that $\omega(0)=D$, $\left.\omega(1)\right|_{V_{i}}=i$ and the coefficients $\omega(n)$ generate a representation of the Virasoro Lie algebra:

$$
[\omega(m), \omega(n)]=(m-n) \omega(m+n-1)+\delta_{m+n, 2} \frac{1}{2}\left(\begin{array}{c}
m-1 \\
3
\end{array}\right) c
$$

for some constant $c \in \mathbb{k}$ called the central charge of $V$. In this case $V$ is called conformal vertex algebra or, when $\operatorname{dim} V_{i}<\infty$, a vertex operator algebra. The condition (2.6) is equivalent to the following relations

$$
\omega(0) \omega=D \omega, \quad \omega(1) \omega=2 \omega, \quad \omega(2) \omega=0, \quad \omega(3) \omega=\frac{c}{2}, \quad \omega(n) \omega=0 \quad \text { for } n \geqslant 4 .
$$

This means that $\omega$ generates a Virasoro conformal algebra, see [14].

Definition 2.2 ([12]). A vertex algebra $V$ is called OZ (abbreviation of "One-Zero") if it is graded so that $V=\mathbb{k} \mathbb{1} \oplus \bigoplus_{n \geqslant 2} V_{n}$. 
An OZ vertex algebra $V$ has $\operatorname{dim} V_{0}=1$ and $\operatorname{dim} V_{1}=0$, which explains the name. The component $V_{2}$ is a commutative (but not necessarily associative) algebra with respect to the product $a \otimes b \mapsto a b=a(1) b$, called the Griess algebra of $V$. The commutativity follows from (2.4). The algebra $V_{2}$ has a bilinear form $\langle a \mid b\rangle=a(3) b$. From (2.4) it follows that this form is symmetric, and from (2.5) it follows that it it invariant: $\langle a b \mid c\rangle=\langle a \mid b c\rangle$.

Remark 2.2. It should be noted that the idea that the 196883 -dimensional Griess's algebra can be realized (after adjoining a unit) as a degree 2 component of a vertex algebra is due to Frenkel, Lepowsky and Meurman [9]. The general fact that degree 2 component of any OZ vertex algebra has a commutative algebra structure with a symmetric invariant bilinear form is mentioned in this book as a triviality.

One can define analogous structure on the components $V_{0}$ and $V_{1}$. Namely, if a graded vertex algebra $V$ satisfies $V_{n}=0$ for $n \leqslant 0$, then $V_{0}$ is an associative commutative algebra with respect to the product $(-1)$, and $V_{1}$ is a Lie algebra with respect to the product (0) with an invariant symmetric bilinear form given by the product (1).

We note that all definitions in this subsection make sense for $\mathbb{k}$ being a commutative associative algebra containing $\mathbb{Q}$. In this case by "linear space" we understand a torsion-free $k$-module. This remark applies also to Sections 2.4-2.6 below, and to the parts of Section 2.3 that does not refer to correlation functions.

\subsection{Correlation functions}

Denote by $\Phi^{l}$ the space of rational functions in the variables $z_{1}, \ldots, z_{l}$ of the form

$$
\alpha=p\left(z_{1}, \ldots, z_{l}\right) \prod_{1 \leqslant i<j \leqslant l}\left(z_{i}-z_{j}\right)^{k_{i j}}, \quad p \in \mathbb{k}\left[z_{1}, \ldots, z_{l}\right],
$$

where $k_{i j} \in \mathbb{Z}$. Obviously we have a product $\Phi^{l} \otimes \Phi^{m} \rightarrow \Phi^{l+m}$ given by multiplying the functions and renaming the variables.

Denote by $\operatorname{ord}_{i j} \alpha$ the order of $\alpha \in \Phi^{l}$ at $z_{i}-z_{j}$. The space $\Phi^{l}=\bigoplus_{d \in \mathbb{Z}} \Phi_{d}^{l}$ is graded in the usual sense, so that $\operatorname{deg} z_{i}=1$.

Let $V$ be a graded vertex algebra, and let $f: V \rightarrow \mathbb{k}$ be a linear functional of degree $d \in \mathbb{Z}$, i.e. $f\left(V_{n}\right)=0$ for $n \neq d$. Take some elements $a_{1}, \ldots, a_{l} \in V$ of degrees $d_{1}, \ldots, d_{l}$ respectively and formal variables $z_{1}, \ldots, z_{l}$. Consider the series

$$
f\left(Y\left(a_{1}, z_{1}\right) \cdots Y\left(a_{l}, z_{l}\right) \mathbb{1}\right)=\sum_{m_{1}, \ldots, m_{l} \in \mathbb{Z}} f\left(a_{1}\left(m_{1}\right) \cdots a_{l}\left(m_{l}\right) \mathbb{1}\right) z_{1}^{-m_{1}-1} \cdots z_{l}^{-m_{l}-1} .
$$

The following properties of the series (2.9) can be deduced from Definition 2.1 (see [7]):

Rationality. The series (2.9) converge in the domain $\left|z_{1}\right|>\cdots>\left|z_{l}\right|$ to a rational function $\alpha_{f}\left(z_{1}, \ldots, z_{l}\right) \in \Phi^{l}$ such that $\operatorname{ord}_{i j} \alpha_{f} \geqslant-\operatorname{loc}\left(a_{i}, a_{j}\right)$. It is called a correlation function of $V$.

Commutativity. For any permutation $\sigma \in \Sigma_{l}$, the correlation function corresponding to $a_{\sigma(1)}, \ldots, a_{\sigma(l)}$ and the same functional $f: V \rightarrow \mathbb{k}$ is $\alpha_{f}\left(z_{\sigma(1)}, \ldots, z_{\sigma(l)}\right)$.

Associativity. The series

$$
f\left(Y\left(Y\left(a_{1}, z_{1}-z_{2}\right) a_{2}, z_{2}\right) Y\left(a_{3}, z_{3}\right) \cdots Y\left(a_{l}, z_{l}\right) \mathbb{1}\right)
$$

converge in the domain $\left|z_{2}\right|>\cdots>\left|z_{l}\right|>\left|z_{1}-z_{2}\right|>0$ to $\alpha_{f}\left(z_{1}, \ldots, z_{l}\right)$. 
Since $\operatorname{deg} a_{i}\left(m_{i}\right)=d_{i}-m_{i}-1$, we get $\operatorname{deg} \alpha_{f}=\operatorname{deg} f-\sum_{i=1}^{l} d_{i}$.

It can be shown (see $[7,17]$ ) that the rationality and commutativity properties of correlation functions together with the conditions (2.2) and (2.3) can serve as an equivalent definition of vertex algebras. We will use this fact in Section 4 below.

In order to explain the meaning of the associativity condition, we need to introduce another definition. Take some $1 \leqslant i<j \leqslant l$. A function $\alpha \in \Phi^{l}$ has expansion

$$
\alpha\left(z_{1}, \ldots, z_{n}\right)=\sum_{k \geqslant k_{0}} \alpha_{k}\left(z_{1}, \ldots, \widehat{z_{i}}, \ldots, z_{l}\right)\left(z_{i}-z_{j}\right)^{k}
$$

for $\alpha_{k} \in \Phi^{l-1}$. Here and below the hat over a term indicates that this term is omitted. Then we define the operators $\rho_{i j}^{(k)}: \Phi^{l} \rightarrow \Phi^{l-1}$ by setting

$$
\rho_{i j}^{(k)} \alpha=\alpha_{k}
$$

An important property of these maps is that for any $1 \leqslant i<j \leqslant l$ and $1 \leqslant s<t \leqslant l$, such that $\{s, t\} \cap\{i, j\}=\varnothing$, and $m, k \in \mathbb{Z}$,

$$
\rho_{s t}^{(m)} \rho_{i j}^{(k)}=\rho_{i j}^{(k)} \rho_{s t}^{(m)} .
$$

Now the associativity condition means that if $\alpha_{f}$ is a correlation function corresponding to the elements $a_{1}, \ldots, a_{l}$ and a functional $f: V \rightarrow \mathbb{k}$, then $\rho_{12}^{(k)} \alpha_{f}$ is the correlation function corresponding to the elements $a_{1}(-k-1) a_{2}, a_{3}, \ldots, a_{l}$ and the same functional $f$.

\subsection{The action of $s l_{2}$}

In this paper we will deal with vertex algebras equipped a certain action of the Lie algebra $s l_{2}$.

Definition 2.3. A vertex algebra $V$ is said to have $s l_{2}$ structure, if $V=\bigoplus_{d \in \mathbb{Z}} V_{d}$ is graded, and there is a locally nilpotent operator $D^{*}: V \rightarrow V$ of degree -1 , such that $D^{*} \mathbb{1}=0$ and

$$
\left[D^{*}, a(m)\right]=(2 d-m-2) a(m+1)+\left(D^{*} a\right)(m)
$$

for every $a \in V_{d}$.

Let $\delta: V \rightarrow V$ be the grading derivation, defined by $\left.\delta\right|_{V_{d}}=d$. It is easy to compute that if $D^{*}: V \rightarrow V$ satisfies condition (2.13), then

$$
\left[D^{*}, D\right]=2 \delta, \quad[\delta, D]=D, \quad\left[\delta, D^{*}\right]=-D^{*},
$$

so that $D^{*}, D$ and $\delta$ span a copy of $s l_{2}$.

All vertex algebras in this paper are assumed to have $s l_{2}$ structure, all ideals are stable under $s l_{2}$ and homomorphisms of vertex algebras preserve the action of $s l_{2}$.

An element $a \in V$ such that $D^{*} a=0$ is called minimal. It is easy to see that if $V$ is generated by minimal elements, then any operator $D^{*}: V \rightarrow V$ satisfying (2.13) must be locally nilpotent.

If $V$ has a Virasoro element $\omega$, then we can take $D^{*}=\omega(2)$. Note that we always have $D=\omega(0)$ and $\delta=\omega(1)$, therefore conformal vertex algebras always have an $s l_{2}$ structure.

Vertex algebras with an action of $s l_{2}$ as above were called quasi-vertex operator algebras in [7] and homogeneous minimal elements are sometimes called quasi-primary.

Now we describe the dual action on the correlation functions. It follows from (2.3) that the operator dual to $D$ is $\Delta=\partial_{z_{1}}+\cdots+\partial_{z_{l}}$, so that

$$
f\left(D Y\left(a_{1}, z_{1}\right) \cdots Y\left(a_{l}, z_{l}\right) \mathbb{1}\right)=\Delta \alpha_{f}\left(z_{1}, \ldots, z_{l}\right)
$$


for any homogeneous $a_{1}, \ldots, a_{l} \in V$ and $f: V \rightarrow \mathbb{k}$. Note that $\Delta: \Phi^{l} \rightarrow \Phi^{l}$ is an operator of degree -1 .

To describe the dual operator of $D^{*}$, consider the differential operator $\Delta^{*}(n, z)=z^{2} \partial_{z}+n z$. For the formal variables $z_{1}, \ldots, z_{l}$, and for a sequence of integers $n_{1}, \ldots, n_{l}$ set

$$
\Delta^{*}\left(n_{1}, \ldots, n_{l}\right)=\Delta^{*}\left(n_{1}, z_{1}\right)+\cdots+\Delta^{*}\left(n_{l}, z_{l}\right) .
$$

By (2.13), we have

$$
f\left(D^{*} Y\left(a_{1}, z_{1}\right) \cdots Y\left(a_{l}, z_{l}\right) \mathbb{1}\right)=\Delta^{*}\left(2 d_{1}, \ldots, 2 d_{l}\right) \alpha_{f}\left(z_{1}, \ldots, z_{l}\right)
$$

for minimal homogeneous elements $a_{1}, \ldots, a_{l} \in V$ of degrees $\operatorname{deg} a_{i}=d_{i}$ and a functional $f$ : $V \rightarrow \mathbb{k}$.

Using the relations

$$
\begin{aligned}
& \Delta^{*}\left(n_{1}, \ldots, n_{l}\right)\left(z_{i}-z_{j}\right)=\left(z_{i}-z_{j}\right) \Delta^{*}\left(n_{1}, \ldots, n_{i}+1, \ldots, n_{j}+1, \ldots, n_{l}\right), \\
& \Delta^{*}\left(n_{1}, \ldots, n_{l}\right) z_{i}=z_{i} \Delta^{*}\left(n_{1}, \ldots, n_{i}+1, \ldots, n_{l}\right),
\end{aligned}
$$

where $z_{i}$ and $z_{i}-z_{j}$ are viewed as operators on $\Phi^{l}$, we see that $\Delta^{*}\left(n_{1}, \ldots, n_{l}\right)$ is an operator on $\Phi^{l}$ of degree 1 .

We are going to need some easy facts about $s l_{2}$-module structure of $V$ :

\section{Lemma 2.1.}

a. If $d<0$, then $V_{d}=\left(D^{*}\right)^{1-d} V_{1}$.

b. $D V_{-1} \subseteq D^{*} V_{1}$.

These statements hold for any graded $s l_{2}$-module $V$ on which $D^{*}$ is locally nilpotent and $\left.\delta\right|_{V_{d}}=d[24]$. The second statement follows easily from the first:

$$
D V_{-1}=D D^{*} V_{0}=D^{*} D V_{0} \subseteq D^{*} V_{1}
$$

For vertex algebras the action of $s l_{2}$ was also investigated in [5].

\subsection{The universal enveloping algebra}

For any vertex algebra $V$ we can construct a Lie algebra $L=$ Coeff $V$ in the following way $[1,14,20,22]$. Consider the linear space $\mathbb{k}\left[t, t^{-1}\right] \otimes V$, where $t$ is a formal variable. Denote $a(n)=a \otimes t^{n}$ for $n \in \mathbb{Z}$. As a linear space, $L$ is the quotient of $\mathbb{k}\left[t, t^{-1}\right] \otimes V$ by the subspace spanned by the relations $(D a)(n)=-n a(n-1)$. The brackets are given by

$$
[a(m), b(n)]=\sum_{i \geqslant 0}\left(\begin{array}{c}
m \\
i
\end{array}\right)(a(i) b)(m+n-i),
$$

which is precisely the identity (V4) of Definition 2.1. The spaces $L_{ \pm}=\operatorname{Span}\{a(n) \mid n \geqslant 0\} \subset L$ are Lie subalgebras of $L$ so that $L=L_{-} \oplus L_{+}$.

Remark 2.3. The construction of $L$ makes use of only the products $(n)$ for $n \geqslant 0$ and the map $D$. This means that it works for a more general algebraic structure, known as conformal algebra $[14,21]$. 
Now assume that the vertex algebra $V$ has an $s l_{2}$ structure. Then (V3) of Definition 2.1 and (2.13) define derivations $D: L \rightarrow L$ and $D^{*}: L \rightarrow L$ so we get an action of $s l_{2}$ on $L$ by derivations. Denote by $\widehat{L}=L \rtimes s l_{2}$ the corresponding semi-direct product.

The Lie algebra $\widehat{L}=\widehat{\text { Coeff } V}$ and its universal enveloping algebra $U=U(\widehat{L})$ inherit the grading from $V$ so that $\operatorname{deg} a(m)=\operatorname{deg} a-m-1$. The Frenkel-Zhu topology [10] on a homogeneous component $U_{d}$ is defined by setting the neighborhoods of 0 to be the spaces $U_{d}^{k}=\sum_{i \leqslant k} U_{d-i} U_{i}$, so that

$$
\cdots \subset U_{d}^{k-1} \subset U_{d}^{k} \subset U_{d}^{k+1} \subset \cdots \subset U_{d}, \quad \bigcap_{k \in \mathbb{Z}} U_{d}^{k}=0, \quad \bigcup_{k \in \mathbb{Z}} U_{d}^{k}=U_{d} .
$$

Let $\bar{U}=\bigoplus_{d \in \mathbb{Z}} \bar{U}_{d}$ be the completion of $U(\widehat{L})$ in this topology. Consider the ideal $I \subset \bar{U}$ generated by the relations

$$
(a(m) b)(n)=\sum_{s \geqslant 0}(-1)^{s}\left(\begin{array}{c}
m \\
s
\end{array}\right) a(m-s) b(n+s)-\sum_{s \leqslant m}(-1)^{s}\left(\begin{array}{c}
m \\
m-s
\end{array}\right) b(n+s) a(m-s)
$$

for all $a, b \in V$ and $m, n \in \mathbb{Z}$. Note that the relations above are simply the associativity identity (2.5). Denote by $W=\bar{U} / \bar{I}$ the quotient of $\bar{U}$ by the closure of $I$.

For a finite ordered set of elements $\mathcal{S}=\left\{a_{1}, \ldots, a_{l}\right\}, a_{i} \in V$, let $W_{\mathcal{S}}$ be the $\left\langle D, \delta, D^{*}\right\rangle$-module generated by all monomials $a_{1}\left(m_{1}\right) \cdots a_{l}\left(m_{l}\right) \in W, m_{i} \in \mathbb{Z}$.

Definition 2.4 ([10, 24]). The universal enveloping algebra of $V$ is

$$
U(V)=\bigcup_{\mathcal{S}} \bar{W}_{\mathcal{S}} \subset W
$$

where the union is taken over all finite ordered sets $\mathcal{S} \subset V$, and $\bar{W}_{\mathcal{S}} \subset W$ is the completion of the space $W_{\mathcal{S}}$ in the Frenkel-Zhu topology.

Remark 2.4. In fact, it follows from the commutativity property of correlation functions (see Section 2.2) that if $\mathcal{S}$ and $\mathcal{S}^{\prime}$ differ by a permutation, then $\bar{W}_{\mathcal{S}}=\bar{W}_{\mathcal{S}^{\prime}}$.

It is proved in [24] that any module over a vertex algebra $V$ is a continuous module over $U(V)$, in the sense that for any sequence $u_{1}, u_{2}, \ldots \in U(V)$ that converges to 0 and for any $v \in M$ we have $u_{i} v=0$ for $i \gg 0$. Conversely, any $U(V)$-module $M$, such that $a(m) v=0$ for any $a \in V$, $v \in M$ and $a(m) v=0$ for $m \gg 0$, is a module over $V$.

Remark 2.5. The algebra $W=\bar{U}(\widehat{L}) / \bar{I}$ is also a good candidate for universal enveloping algebra of $V$. It has the following property [10]: consider a graded space $M$ such that $M_{d}=0$ for $d \ll 0$; then $M$ is a $W$-module if and only if $M$ is an $V$-module.

On the other hand, we could define an algebra $\widehat{U}(V)$ such that any series of elements from $U(\widehat{L})$, that make sense as an operator on any $V$-module, would converge in $\widehat{U}(V)$. However, this algebra is too big for our purposes, for example there is no way of defining an involution in this algebra, as we do in Section 2.5 below.

\subsection{Invariant bilinear forms}

The key ingredient of our constructions is the notion of invariant bilinear form on vertex algebra. Here we review the results of [24], that generalize the results of Frenkel, Huang and Lepowsky [7] and Li [18]. 
Let $V$ be a vertex algebra with an $s l_{2}$ structure, as in Section 2.3. It is shown in $[7,24]$, that there is an anti-involution $u \mapsto u^{*}$ on the universal enveloping algebra $U(V)$ such that $D \mapsto D^{*}$, $D^{*} \mapsto D, \delta^{*}=\delta$ and

$$
a(m)^{*}=(-1)^{\operatorname{deg} a} \sum_{i \geqslant 0} \frac{1}{i !}\left(\left(D^{*}\right)^{i} a\right)(2 \operatorname{deg} a-m-2-i)
$$

for a homogeneous $a \in V$ and $m \in \mathbb{Z}$. In particular, if $D^{*} a=0$, then

$$
a(m)^{*}=(-1)^{\operatorname{deg} a} a(2 \operatorname{deg} a-m-2),
$$

which can be written as

$$
Y(a, z)^{*}=\sum_{m \in \mathbb{Z}} a(m)^{*} z^{-m-1}=(-1)^{\operatorname{deg} a} Y\left(a, z^{-1}\right) z^{-2 \operatorname{deg} a} .
$$

It is proved in [24] that for any $u \in U(V)_{0}$,

$$
u \mathbb{1}-u^{*} \mathbb{1} \in D^{*} V_{1} .
$$

Let $K$ be a linear space over $\mathbb{k}$.

Definition $2.5([7,18])$. A $K$-valued bilinear form $\langle\cdot \mid \cdot\rangle$ on $V$ is called invariant if

$$
\langle a(m) b \mid c\rangle=\left\langle b \mid a(m)^{*} c\right\rangle \quad \text { and } \quad\langle D a \mid b\rangle=\left\langle a \mid D^{*} b\right\rangle
$$

for all $a, b, c \in V$ and $m \in \mathbb{Z}$.

The radical $\operatorname{Rad}\langle\cdot \mid \cdot\rangle=\{a \in V \mid\langle a \mid b\rangle=0 \forall b \in V\}$ of an invariant form is an ideal of $V$. Also, since $\langle\delta a \mid b\rangle=\langle a \mid \delta b\rangle$, we have $\left\langle V_{i} \mid V_{j}\right\rangle=0$ for $i \neq j$.

Given a $K$-valued invariant form $\langle\cdot \mid \cdot\rangle$ on $V$, one can consider a linear functional $f: V_{0} \rightarrow K$ defined by $f(a)=\langle\mathbb{1} \mid a\rangle$. Since $f\left(D^{*} a\right)=\left\langle\mathbb{1} \mid D^{*} a\right\rangle=\langle D \mathbb{1} \mid a\rangle=0$, we get that $f\left(D^{*} V_{1}\right)=0$. Also, the form can be reconstructed from $f$ by the formula $\langle a \mid b\rangle=f\left(a(-1)^{*} b\right)$.

Proposition 2.1 ([18, 24]). There is a one-to-one correspondence between invariant $K$-valued bilinear forms $\langle\cdot \mid \cdot\rangle$ on a vertex algebra $V$ and linear functionals $f: V_{0} / D^{*} V_{1} \rightarrow K$, given by $f(a)=\langle\mathbb{1} \mid a\rangle,\langle a \mid b\rangle=f\left(a(-1)^{*} b\right)$. Moreover, every invariant bilinear form on $V$ is symmetric.

Remark 2.6. We observe that a vertex algebra $V$ such that $V_{0}=\mathbb{k} \mathbb{1}$ and $D^{*} V_{1}=0$ is simple if and only if the invariant $\mathrm{k}$-valued bilinear form on $V$ (which is unique by the above) is nondegenerate. Indeed, any homomorphism $V \rightarrow U$ of vertex algebras must be an isometry, hence its kernel must belong to the radical of the form.

\subsection{Radical of a vertex algebra}

Let $I=\left\langle D^{*} V_{1}\right\rangle \subset V$ be the ideal of a vertex algebra $V$ generated by the space $D^{*} V_{1}$. Its degree 0 component $I_{0}=U(V)_{0} D^{*} V_{1}$ is spanned by the elements $a_{1}\left(m_{1}\right) \cdots a_{l}\left(m_{l}\right) D^{*} v$ such that $a_{i} \in V$, $m_{i} \in \mathbb{Z}, \operatorname{deg} a_{1}\left(m_{1}\right) \cdots a_{l}\left(m_{l}\right)=0$ and $v \in V_{1}$, since we have $D V_{-1} \subset D^{*} V_{1} \subset I_{0}$ by Lemma 2.1b. Note that Lemma 2.1a also implies that $V_{d} \subset I$ for $d<0$.

It follows from (2.4) and (2.5) that $K=V_{0} / I_{0}$ is the commutative associative algebra with respect to the product $(-1)$ with unit $\mathbb{1}$. Let $f: V_{0} \rightarrow K$ be the canonical projection. By Proposition 2.1, the map $f$ corresponds to an invariant $K$-valued bilinear form $\langle\cdot \mid \cdot\rangle$ on $V$.

Definition 2.6. The radical of $V$ is $\operatorname{Rad} V=\operatorname{Rad}\langle\cdot \mid \cdot\rangle$. 
Remark 2.7. This definition has nothing to do with the radical defined in [4].

Denote $\bar{V}=V / \operatorname{Rad} V$. The following proposition summarizes some properties of $\bar{V}$ that we will need later.

Proposition $2.2([24])$.

a. $\operatorname{Rad}(\bar{V})=0$.

b. $\bar{V}=\bigoplus_{n \geqslant 0} \bar{V}_{n}$, so that $\bar{V}_{0}=V_{0} / I_{0}=K$, and $\bar{V}$ is a vertex algebra over $K$.

c. Every ideal $J_{0} \subset K$ can be canonically extended to an ideal $J \subset \bar{V}$, such that $J \cap \bar{V}_{0}=J_{0}$. The ideal $J$ is the maximal among all ideals $I \subset \bar{V}$ with the property $I \cap \bar{V}_{0}=J_{0}$. In particular there are no non-trivial ideals $I \subset \bar{V}$ such that $I \cap \bar{V}_{0}=0$.

The ideal $J \subset \bar{V}$ extending $J_{0} \subset \bar{V}_{0}$ is constructed in the following way: let $g: K \rightarrow K / J_{0}$ be the canonical projection, by Proposition 2.1 it defines a $K / J_{0}$-valued invariant bilinear form $\langle\cdot \mid \cdot\rangle_{g}$ on $\bar{V}$. Then set $J=\operatorname{Rad}\langle\cdot \mid \cdot\rangle_{g}$.

\section{Regular functions}

\subsection{Components}

Let $V$ be a vertex algebra with $s l_{2}$ structure. As in Section 2.2, take some homogeneous elements $a_{1}, \ldots, a_{l} \in V$ of $\operatorname{deg} a_{i}=d_{i}$ and a functional $f: V_{d} \rightarrow \mathbb{k}$ of degree $\mathrm{d}$, and let $\alpha=\alpha_{f}\left(z_{1}, \ldots, z_{l}\right) \in$ $\Phi^{l}$ be the corresponding correlation function, given by (2.9). We have $\operatorname{deg} \alpha=d-\sum_{i} d_{i}$.

Denote by $\mathcal{P}$ the set of all partitions $\{1, \ldots, l\}=I \sqcup J$ of the set $\{1, \ldots, l\}$ into two disjoint subsets. For every $P=(I, J) \in \mathcal{P}$, the function $\alpha$ has an expansion

$$
\alpha=\sum_{n \geqslant m}(\alpha)_{n}, \quad \text { where } \quad(\alpha)_{n}=(\alpha)_{n}(P)=\sum_{j} \alpha_{d-n, j}^{\prime} \alpha_{n, j}^{\prime \prime},
$$

for some $m \in \mathbb{Z}$. This expansion is obtained in the following way: Let $I=\left\{i_{1}, \ldots, i_{|I|}\right\}$ and $J=\left\{j_{1}, \ldots, j_{|I|}\right\}$. Expand $\alpha$ in power series in the domain $\left|z_{i_{1}}\right|>\left|z_{i_{2}}\right|>\cdots>\left|z_{j_{1}}\right|>\left|z_{j_{2}}\right|>\cdots$ and collect terms with powers of $\left\{z_{i} \mid i \in I\right\}$ and $\left\{z_{j} \mid j \in J\right\}$. Note that the second sum in (3.1) is finite. Here $\alpha_{n, j}^{\prime}$ and $\alpha_{n, j}^{\prime \prime}$ are rational functions depending on the variables $\left\{z_{i} \mid i \in I\right\}$, and $\alpha_{n, j}^{\prime \prime}$ and $\left\{z_{i} \mid i \in J\right\}$ respectively, and we have

$$
\operatorname{deg} \alpha_{n, j}^{\prime}=n-\sum_{i \in I} d_{i} \quad \text { and } \quad \operatorname{deg} \alpha_{n, j}^{\prime \prime}=n-\sum_{i \in J} d_{i}
$$

We call the term $(\alpha)_{n}$ in (3.1) the component of $\alpha$ of degree $n$ corresponding to partition $I \sqcup J$. Note that $\alpha_{n, j}^{\prime \prime} \in \Phi^{|J|}$, while in general $\alpha_{n, j}^{\prime} \notin \Phi^{|I|}$, since $\alpha_{n, j}^{\prime}$ may have a pole at $z_{i}$.

Assume that $\alpha=\alpha_{f}$ satisfies $(\alpha)_{n}=0$ for $n<m$, and assume that $I=\left\{i_{1}, \ldots, i_{r}\right\}$, $J=\left\{i_{r+1}, \ldots, i_{l}\right\}$. Then $f\left(a_{i_{1}}\left(m_{1}\right) \cdots a_{i_{l}}\left(m_{l}\right) \mathbb{1}\right)=0$ whenever $\operatorname{deg} a_{i_{r+1}} \cdots a_{i_{l}}\left(m_{l}\right)<m$. For example, suppose that $k$ is the order of $\alpha$ at $z_{i}=z_{j}$. Take a partition $\{1, \ldots, l\}=$ $\{1, \ldots, \widehat{i}, \ldots, \widehat{j}, \ldots, l\} \sqcup\{i, j\}$. Then $(\alpha)_{n}=0$ for $n<d_{i}+d_{j}+k$, due to the associativity property of Section 2.2 .

We are going to use the above terminology even when $\alpha \in \Phi^{l}$ does not necessarily correspond to a linear functional on a vertex algebra (for some fixed integers $k_{1}, \ldots, k_{l}$ ). 


\subsection{Components of degree 0}

Denote by $\overline{\mathcal{P}}$ the set of unordered partitions of $\{1, \ldots, l\}$. Clearly, we have a projection $\mathcal{P} \ni$ $P \mapsto \bar{P} \in \overline{\mathcal{P}}$.

Fix some integers $d_{1}, \ldots, d_{l}$. Suppose that a function $\alpha \in \Phi^{l}$ of degree $-\sum d_{i}$ satisfies $(\alpha)_{n}(P)=0$ for all $n<0$ and $P \in \mathcal{P}$. Then the expansion (3.1) has a leading term $(\alpha)_{0}(P)$. It is easy to see that $(\alpha)_{0}$ depends only on the unordered partion $\bar{P}$.

Proposition 3.1. Suppose that for every partition $P=\left(I_{1}, I_{2}\right) \in \overline{\mathcal{P}}$ we have a function $\alpha(P)=\sum_{j} \alpha_{j}^{(1)} \alpha_{j}^{(2)}$, where $\alpha_{j}^{(s)}$ depends on the variables $\left\{z_{i} \mid i \in I_{s}\right\}$, $\operatorname{deg} \alpha_{j}^{(s)}=-\sum_{i \in I_{s}} d_{i}$ and $\left(\alpha_{j}^{(s)}\right)_{d}=0$ for $d<0, s=1,2$. Assume that for any $Q \in \overline{\mathcal{P}}$ we have

$$
(\alpha(P))_{0}(Q)=(\alpha(Q))_{0}(P) .
$$

Then there is a function $\alpha \in \Phi^{l}, k_{P} \in \mathbb{k}$, such that $(\alpha)_{0}(P)=\alpha(P)$. Moreover, $\alpha$ is a linear combination of $\alpha(P)$ 's and their degree 0 components.

Proof. Introduce a linear ordering on the subsets of $\{1, \ldots, l\}$ such that $I<J$ if $|I|<|J|$, and then extend it to $\overline{\mathcal{P}}$ so that $P=\left\{I_{1}, I_{2}\right\}<Q=\left\{J_{1}, J_{2}\right\}$ if $I_{1}<J_{1}$ and $I_{1} \leqslant I_{2}, J_{1} \leqslant J_{2}$. Set $P_{\text {min }}=\min \{P \in \overline{\mathcal{P}} \mid \alpha(P) \neq 0\}$. We will prove the existence of $\alpha$ by induction on $\mid\{P \in$ $\overline{\mathcal{P}} \mid \alpha(P) \neq 0\} \mid$. If $\alpha(P)=0$ for all $P$, take $\alpha=0$.

We observe that if $\alpha \in \Phi^{l}$ has degree $-\sum_{i} d_{i}$ and $(\alpha)_{d}=0$ for $d<0$, then the family of components $\left\{\alpha(P)=(\alpha)_{0}(P)\right\}_{P \in \overline{\mathcal{P}}}$ satisfies (3.2). Also, if collections $\{\alpha(P)\}$ and $\{\beta(P)\}$ satisfy (3.2), then so does $\{\alpha(P)+\beta(P)\}$.

Now for any $P \in \overline{\mathcal{P}}_{2}$ set $\beta(P)=\alpha(P)-\left(\alpha\left(P_{\text {min }}\right)\right)_{0}(P)$. Obviously, $\beta\left(P_{\min }\right)=0$, and also, if $P<P_{\min }$, then $\alpha(P)=0$ and hence, using $(3.2), \beta(P)=-\left(\alpha\left(P_{\min }\right)_{0}\right)(P)=-\left(\alpha(P)_{0}\right)\left(P_{\min }\right)=0$. By the above observation, the collection $\{\beta(P)\}$ satisfies (3.2), therefore by induction, there is a function $\beta \in \Phi^{l}$, such that $(\beta)_{0}(P)=\beta(P)$ for any $P \in \overline{\mathcal{P}}$. Now take $\alpha=\beta+\alpha\left(P_{\min }\right)$.

Remark 3.1. We can define components $(\alpha)_{n}(P)$ and the decomposition (3.1) for partitions $P$ of $\{1, \ldots, l\}$ into more than two parts. Suppose we know the components $\alpha(P)$ for all such partitions $P$. Then one can show that the function $\alpha \in \Phi^{l}$, such that $(\alpha)_{0}(P)=\alpha(P)$, can be reconstructed by the following formula:

$$
\alpha=\sum_{P \in \overline{\mathcal{P}}}(-1)^{|P|}(|P|-1) ! \alpha(P),
$$

where $\overline{\mathcal{P}}$ is the set of all unordered partitions of $\{1, \ldots, l\}$ and $|P|$ is the number of parts of a partition $P \in \overline{\mathcal{P}}$.

Remark 3.2. It follows from the proof of Proposition 3.1 that instead of (3.2) it is enough to require that the components $\alpha(P)$ satisfy the following property: If $\alpha(Q)=0$ for some $Q \in \overline{\mathcal{P}}$, then $(\alpha(P))_{0}(Q)=0$ for every $P \in \overline{\mathcal{P}}$.

\subsection{Regular functions}

Recall that in Section 2.3 we have defined operators $\Delta$ and $\Delta^{*}$, so that for a correlation function $\alpha_{f}\left(z_{1}, \ldots, z_{n}\right) \in \Phi^{l}$ corresponding to a linear functional $f: V_{d} \rightarrow \mathbb{k}$ and elements $a_{1} \in V_{d_{1}}, \ldots, a_{l} \in V_{d_{l}}$ we have $f\left(D V_{d-1}\right)=0$ if and only if $\Delta \alpha_{f}=0$ and $f\left(D^{*} V_{d+1}\right)=0$ if and only if $\Delta^{*}\left(2 d_{1}, \ldots, 2 d_{l}\right) \alpha_{f}=0$.

It is easy to describe all functions $\alpha\left(z_{1}, \ldots, z_{l}\right) \in \Phi^{l}$, such that $\Delta \alpha=0$. These are the functions $\alpha$ that are invariant under translations, since

$$
\alpha\left(z_{1}+t, \ldots, z_{l}+t\right)=\exp (t \Delta) \alpha\left(z_{1}, \ldots, z_{l}\right)=\alpha\left(z_{1}, \ldots, z_{l}\right) .
$$

by the Taylor formula. In other words, such $\alpha$ depends only on the differences $z_{i}-z_{j}$. 
Now we will investigate the functions $\alpha$ which are killed by $\Delta^{*}$.

Definition 3.1. A function $\alpha \in \Phi^{l}$ is called $\left(n_{1}, \ldots, n_{l}\right)$-regular if $\Delta^{*}\left(n_{1}, \ldots, n_{l}\right) \alpha_{f}=0$.

Example 3.1. For an integer symmetric $l \times l$ matrix $\mathrm{S}=\left\{\mathrm{s}_{\mathrm{ij}}\right\}$ with $s_{i i}=0$ define

$$
\pi(S)=\prod_{1 \leqslant i<j \leqslant 1}\left(z_{i}-z_{j}\right)^{s_{i j}} \in \Phi^{\prime} .
$$

The relations (2.14) imply that

$$
\Delta^{*}\left(n_{1}, \ldots, n_{l}\right)(\pi(\mathrm{S}))=\left(\left(\mathrm{n}_{1}+\mathrm{s}_{1}\right) \mathrm{z}_{1}+\cdots+\left(\mathrm{n}_{\mathrm{l}}+\mathrm{s}_{\mathrm{l}}\right) \mathrm{z}_{1}\right) \pi(\mathrm{S}),
$$

where $s_{i}=\sum_{j} s_{i j}$. Therefore, $\Delta^{*}\left(n_{1}, \ldots, n_{l}\right)(\pi(\mathrm{S}))=0$ if and only if $s_{i}=-n_{i}$ for $i=1, \ldots, l$. In this case the matrix $\mathbf{S}$ will be called $\left(n_{1}, \ldots, n_{l}\right)$-regular, so that $\pi(\mathrm{S})$ is a regular function whenever $\mathrm{S}$ is a regular matrix.

Remark 3.3. One can show, though we will not use this here, that the space of regular functions $\operatorname{Ker} \Delta^{*}\left(n_{1}, \ldots, n_{l}\right) \subset \Phi^{l}$ is spanned by the products $\pi(\mathrm{S})$ where $\mathrm{S}=\left\{\mathrm{s}_{\mathrm{ij}}\right\}$ runs over the set of $n_{1}, \ldots, n_{l}$-regular matrices such that $s_{i j} \geqslant k_{i j}$. This description is analogous to the description of Ker $\Delta$ above. Moreover, using this description, the dimensions of the homogeneous components of the spaces $\operatorname{Ker} \Delta^{*}\left(n_{1}, \ldots, n_{l}\right) \subset \Phi^{l}$ can be given a combinatorial interpretation, in fact, they are certain generalizations of Catalan numbers.

Assume we have a homogeneous linear functional $f: V \rightarrow \mathbb{k}$ such that $f\left(D^{*} V\right)=0$, and the elements $a_{i} \in V_{d_{i}}, i=1, \ldots, l$. Then by Lemma 2.1 a, we have $\operatorname{deg} f \geqslant 0$, and therefore the corresponding correlation function $\alpha_{f}$, given by $(2.9)$, is $\left(2 d_{1}, \ldots, 2 d_{l}\right)$-regular and satisfies $\operatorname{deg} \alpha_{f} \geqslant-\sum_{i} d_{i}$. Moreover, if $\operatorname{deg} f=0$, then also $f(D V)=0$ by Lemma $2.1 \mathrm{~b}$, and therefore $\Delta \alpha_{f}=0$.

Let us investigate the effect of the anti-involution $u \mapsto u^{*}$ of the enveloping algebra $U(V)$ (see Section 2.5) on the correlation functions. Similarly to the series (2.9), one can consider the series

$$
f\left(\left(Y\left(a_{1}, z_{1}\right) \cdots Y\left(a_{l}, z_{l}\right)\right)^{*} \mathbb{1}\right)=f\left(Y\left(a_{l}, z_{l}\right)^{*} \cdots Y\left(a_{1}, z_{1}\right)^{*} \mathbb{1}\right),
$$

which can be shown to converge in the domain $\left|z_{1}\right|<\cdots<\left|z_{l}\right|$ to a rational function $\alpha_{f}^{*}\left(z_{1}, \ldots, z_{l}\right) \in \Phi^{l}$. Since $f\left(D^{*} a_{i}\right)=0$, we can apply the formula $(2.16)$ to each $a_{i}(n)^{*}$ and then it is easy ti check that

$$
\alpha_{f}^{*}=(-1)^{d_{1}+\cdots+d_{l}} z_{1}^{-2 d_{1}} \ldots z_{l}^{-2 d_{l}} \alpha_{f}\left(z_{1}^{-1}, \ldots, z_{l}^{-1}\right) .
$$

It follows from (2.17) and the fact that $f\left(D^{*} V_{1}\right)=0$ that $\alpha_{f}^{*}=\alpha_{f}$.

In Section 4.3, given a collection of integers $\left\{k_{i j}\right\}$ for $1 \leqslant i<j \leqslant l$, we will construct a vertex algebra $F$ such that any function $\alpha \in \Phi^{l}$ such that $\operatorname{ord}_{i j} \alpha \geqslant k_{i j}$ will be a correlation function on $F$, therefore the above properties hold for any $\left(2 d_{1}, \ldots, 2 d_{l}\right)$-regular function. It follows that the above properties of correlation functions hold for all functions in $\Phi^{l}$. Namely, we have the following proposition:

Proposition 3.2. Let $\alpha \in \Phi^{l}$ be a $\left(2 d_{1}, \ldots, 2 d_{l}\right)$-regular function. Then $\operatorname{deg} \alpha \geqslant-\sum_{i} d_{i}$ and if $\operatorname{deg} \alpha=-\sum_{i} d_{i}$, then $\Delta \alpha=0$ and $\alpha^{*}=\alpha$.

Here $\alpha^{*}$ is given by $(3.5)$.

Remark 3.4. Proposition 3.2 can also be easily deduced from the fact that every regular function is a linear combination of the products $\pi(\mathrm{S})$. Also, one can show that a function $\alpha \in \Phi^{l}$ of degree $-\sum d_{i}$ is $\left(2 d_{1}, \ldots, 2 d_{l}\right)$-regular if and only if $\alpha^{*}=\alpha$. 
Corollary 3.1. Let $\alpha \in \Phi^{l}$ be a $\left(2 d_{1}, \ldots, 2 d_{l}\right)$-regular function of degree $-\sum_{i} d_{i}$, and let $(\alpha)_{n}=$ $\sum_{j} \alpha_{-n, j}^{\prime} \alpha_{n, j}^{\prime \prime}$ be the degree $n$ component (3.1) of $\alpha$ with respect to a partition $\{1, \ldots, l\}=I \sqcup J$. Then the degree $n$ component of $\alpha$ with respect to partition $J \sqcup I$ is $\sum_{j}\left(\alpha_{n, j}^{\prime \prime}\right)^{*}\left(\alpha_{-n, j}^{\prime}\right)^{*}$.

Remark 3.5. It is easy to compute using (2.16) that for any correlation function $\alpha$ (and therefore, for any function $\alpha \in \Phi^{l}$ ) one has

$$
\Delta^{*}\left(n_{1}, \ldots, n_{l}\right) \alpha^{*}=-(\Delta \alpha)^{*}
$$

where

$$
\alpha^{*}\left(z_{1}, \ldots, z_{l}\right)=z_{1}^{-n_{1}} \ldots z_{l}^{-n_{l}} \alpha\left(z_{1}^{-1}, \ldots, z_{l}^{-1}\right) .
$$

As before, this can be easily computed without any reference to vertex algebras.

\subsection{Admissible functions}

In this section by "regular" we mean $(4, \ldots, 4)$-regular, and set $\Delta^{*}=\Delta^{*}(4, \ldots, 4)$.

Definition 3.2. A regular function $\alpha \in \Phi^{l}$ is called admissible if for every partition $\{1, \ldots, l\}=$ $I \sqcup J$ we have $(\alpha)_{n}=0$ for $n<0$ or $n=1$. If also $(\alpha)_{0}=0$ for all partitions, then $\alpha$ is called indecomposable.

Denote the space of all admissible functions in $l$ variables by $R^{l} \subset \Phi^{l}$, and the space of all indecomposable admissible functions by $R_{0}^{l} \subset R^{l}$.

We have $R^{1}=0, R^{2}=\mathbb{k}_{(}\left(z_{1}-z_{2}\right)^{-4}, R^{3}=R_{0}^{3}=\mathbb{k}\left(z_{1}-z_{2}\right)^{-2}\left(z_{1}-z_{3}\right)^{-2}\left(z_{2}-z_{3}\right)^{-2}$, and it is easy to compute, using e.g. the representation of regular functions by the products $\pi(\mathrm{S})$, that $\operatorname{dim} R_{0}^{4}=3, \operatorname{dim} R^{4}=6, \operatorname{dim} R_{0}^{5}=16, \operatorname{dim} R^{5}=26$ (compare with Section 5.4 below).

We establish here a few simple properties of admissible functions. Recall that the operators $\rho_{i j}^{(k)}: \Phi^{l} \rightarrow \Phi^{l-1}$ where defined in (2.11).

Proposition 3.3. Let $\alpha \in R^{l}, l \geqslant 3$ and $1 \leqslant i<j \leqslant l$.

a. $\operatorname{ord}_{i j} \alpha \geqslant-4$ and if $\alpha \in R_{0}^{l}$, then $\operatorname{ord}_{i j} \alpha \geqslant-2$.

b. $\rho_{i j}^{(-4)} \alpha \in R^{l-2}, \rho_{i j}^{(-3)} \alpha=0$ and $\rho_{i j}^{(-2)} \alpha \in R^{l-1}$.

c. The function $\alpha$ can be uniquely written as a linear combination of the products of indecomposable admissible functions.

The product in (c) is understood in terms of the operation $\Phi^{l} \otimes \Phi^{m} \rightarrow \Phi^{l+m}$ defined in Section 2.2 .

Proof. To simplify notations, suppose $(i, j)=(l-1, l)$. Consider the expansion (2.10) for the function $\alpha\left(z_{1}, \ldots, z_{l}\right) \in R^{l}$. If we expand every coefficient $\alpha_{k}\left(z_{1}, \ldots, z_{l-1}\right)$ in the power series in $z_{l-1}$ around 0 , we will get exactly the component expansion (3.1) for the partition $\{1, \ldots, l-2\} \sqcup\{l-1, l\}$. Then the minimal component is

$$
(\alpha)_{k_{0}+4}=\alpha_{k_{0}}\left(z_{1}, \ldots, z_{l-2}, 0\right)\left(z_{l-1}-z_{l}\right)^{k_{0}}
$$

where $k_{0}=\operatorname{ord}_{l-1, l} \alpha$. This shows that $k_{0} \geqslant-4$ and, since $(\alpha)_{1}=0$, we have $k_{0} \neq-3$. Also, if $\alpha$ is indecomposable, then $k_{0} \geqslant 2$, which proves (a).

Now assume that $k_{0}=-4$. Then we have

$$
0=(\alpha)_{1}=\left(\left.\frac{\partial \alpha_{-4}}{\partial z_{l-1}}\right|_{z_{l-1}=0}\right)\left(z_{l-1}\left(z_{l-1}-z_{l}\right)^{-4}\right)+\left(\left.\alpha_{3}\right|_{z_{l-1}=0}\right)\left(z_{l-1}-z_{l}\right)^{-3} .
$$


Therefore, $\alpha_{-4}$ does not depend on $z_{l-1}$ and $\alpha_{3}=0$ since $\alpha_{3} \in \Phi^{l-1}$. Since $\alpha$ does not have components of negative degrees or of degree 1 , neither do $\alpha_{-2}$ and $\alpha_{-4}$. To prove (b) we are left to show that $\alpha_{-2}$ and $\alpha_{-4}$ are regular.

We have just seen that the expansion (2.10) for $\alpha$ has form

$$
\alpha=\alpha_{-4}\left(z_{1}, \ldots, z_{l-2}\right)\left(z_{l-1}-z_{l}\right)^{-4}+\alpha_{-2}\left(z_{1}, \ldots, z_{l-1}\right)\left(z_{l-1}-z_{l}\right)^{-2}+O\left(\left(z_{l}-z_{l-1}\right)^{-1}\right) .
$$

Applying $\Delta^{*}$ to this and using (2.14) we get

$$
0=\Delta^{*} \alpha=\left(\Delta_{2}^{*} \alpha_{-4}\right)\left(z_{l-1}-z_{l}\right)^{-4}+\left(\Delta_{1}^{*} \alpha_{-2}\right)\left(z_{l-1}-z_{l}\right)^{-2}+O\left(\left(z_{l}-z_{l-1}\right)^{-1}\right),
$$

where $\Delta_{s}^{*}=\Delta^{*}(4, \ldots, 4)(l-s$ times $), s=1,2$, which proves regularity of $\alpha_{-2}$ and $\alpha_{-4}$.

The proof of (c) is very similar to the proof of Proposition 3.1. Take a partition $P=\left(I_{1}, I_{2}\right) \in \overline{\mathcal{P}}$. We claim that if $(\alpha)_{0}(P)=\sum_{j} \alpha_{0, j}^{\prime} \alpha_{0, j}^{\prime \prime}$, then $\alpha_{0, j}^{\prime} \in R^{\left|I_{1}\right|}$ and $\alpha_{0, j}^{\prime \prime} \in R^{\left|I_{2}\right|}$. Indeed, we only need to check that $\alpha_{0, j}^{\prime}$ 's and $\alpha_{0, j}^{\prime \prime}$ 's are regular. Denote $\Delta_{s}^{*}=\sum_{i \in I_{s}} \Delta^{*}\left(4, z_{i}\right), s=1,2$ (see Section 2.3). Then

$$
0=\Delta^{*}(\alpha)_{0}(P)=\sum_{j}\left(\Delta_{1}^{*} \alpha_{0, j}^{\prime}\right) \alpha_{0, j}^{\prime \prime}+\sum_{j} \alpha_{0, j}^{\prime}\left(\Delta_{2}^{*} \alpha_{0, j}^{\prime \prime}\right)
$$

Therefore, we see, using induction, that $(\alpha)_{0}(P)$ is a linear combination of products of indecomposable admissible functions. In particular we get $(\alpha)_{0}(P) \in R^{l}$.

Let $\alpha \in R^{l}$. If $(\alpha)_{0}(P)=0$ for every partition $P \in \mathcal{P}_{2}$, then $\alpha \in R_{0}^{l}$. Otherwise, let $P \in \mathcal{P}_{2}$ be the minimal partition for which $(\alpha)_{0}(P) \neq 0$. Then the function $\beta=\alpha-(\alpha)_{0}(P) \in R^{l}$ will satisfy $(\beta)_{0}(Q)=0$ for all partitions $Q \leqslant P$. By induction, $\beta$ is a linear combination of products of indecomposable admissible functions, and hence so is $\alpha$.

Remark 3.6. Alternatively, Proposition 3.3c follows from the formula (3.3) in Section 4.6 below.

Remark 3.7. Suppose $\alpha \in \Phi^{l}$ is such that $\operatorname{ord}_{i j} \alpha=-2$. If $\alpha$ is regular, then so is $\rho_{i j}^{(-2)} \alpha$. Indeed, applying $\Delta^{*}$ to

$$
\alpha=\sum_{k \geqslant-2}\left(z_{i}-z_{j}\right)^{k} \rho_{i j}^{(k)} \alpha,
$$

we get, using (2.14),

$$
0=\Delta^{*} \alpha=\sum_{k \geqslant-2}\left(z_{i}-z_{j}\right)^{k} \Delta^{*}(4, \ldots, 8+2 k, \ldots, 4) \rho_{i j}^{(k)} \alpha
$$

Here $8+2 k$ stands at $i$-th position. The coefficient of $\left(z_{i}-z_{j}\right)^{-2}$ in the right-hand side is $\Delta^{*} \rho_{i j}^{(-2)} \alpha$, which should be equal to 0 . In the same way one can check that if $\operatorname{ord}_{i j} \alpha=-4$, then $\rho_{i j}^{(-4)} \alpha$ is regular.

Note that for $\alpha \in R^{l}$ and $1 \leqslant i<j \leqslant l$ we have $\operatorname{ord}_{i j} \alpha \geqslant-4$ and $\rho_{i j}^{(-3)} \alpha=0$.

\subsection{Admissible functions with prescribed poles}

In Section 6 we will need the following property of admissible functions, which is reminiscent of the Mittag-Leffler's theorem for analytic functions. 
Proposition 3.4. Let $l \geqslant 3$, and suppose that for each $1 \leqslant i<j \leqslant l$ we fix admissible functions $\alpha_{i j}^{(-2)} \in R^{(l-1)}$ and $\alpha_{i j}^{(-4)} \in R^{(l-2)}$ satisfying the following condition: For any $1 \leqslant s<t \leqslant l$, such that $\{s, t\} \cap\{i, j\}=\varnothing$,

$$
\rho_{s t}^{(m)} \alpha_{i j}^{(k)}=\rho_{i j}^{(k)} \alpha_{s t}^{(m)}, \quad m, k=-2,-4 .
$$

Then there exists a function $\alpha \in R^{l}$ such that $\rho_{i j}^{(k)} \alpha=\alpha_{i j}^{(k)}$ for all $1 \leqslant i<j \leqslant l$ and $k=-2,-4$.

Note the similarity of the condition on $\alpha_{i j}^{(k)}$ 's with (2.12).

In order to prove this proposition we need the following Lemma.

Lemma 3.1. Let $\alpha \in R^{l}$ be an admissible function. Then for every $1 \leqslant i \leqslant l$ one can write $\alpha=\sum_{m} \alpha_{m}$ for some admissible functions $\alpha_{m} \in R^{l}$ that satisfy the following properties:

(i) Either $\alpha_{m}=\left(z_{i}-z_{j}\right)^{-4} \beta$ for some $j \neq i$, where $\beta \in R^{l-2}$, or $\operatorname{ord}_{i j} \alpha_{m} \geqslant-2$ for all $j \neq i$ and

$$
\left|\left\{j \in\{1, \ldots, l\} \backslash\{i\} \mid \operatorname{ord}_{i j} \alpha_{m}=-2\right\}\right| \leqslant 2 .
$$

(ii) For any $1 \leqslant s<t \leqslant l$, if $\operatorname{ord}_{s t} \alpha \geqslant-1$, then also $\operatorname{ord}_{s t} \alpha_{m} \geqslant-1$, and if $\operatorname{ord}_{s t} \alpha=-2$, then $\operatorname{ord}_{s t} \alpha_{m} \geqslant-2$.

In fact it will follow from the proof that if $\operatorname{ord}_{i j} \alpha_{m}=\operatorname{ord}_{i k} \alpha_{m}=-2$ for some $j \neq k$, then $\alpha_{m}=\left(z_{i}-z_{j}\right)^{-2}\left(z_{i}-z_{k}\right)^{-2}\left(z_{j}-z_{k}\right)^{-2} \beta$, where $\beta \in R^{l-3}$ does not depend on $z_{i}, z_{j}, z_{k}$. Also, by Proposition 3.3c we can always assume that $\alpha_{m}$ is a product of indecomposable admissible functions.

Proof. We use induction on $l$. If $l=2$ (respectively, 3 ), then $\alpha$ is a multiple of $\left(z_{1}-z_{2}\right)^{-4}$ (respectively, $\left(z_{1}-z_{2}\right)^{-2}\left(z_{1}-z_{3}\right)^{-2}\left(z_{2}-z_{3}\right)^{-2}$ ) and we take $\alpha=\alpha_{1}$. So assume that $l \geqslant 4$.

To simplify notations, assume that $i=1$. We also use induction on the number of multiple poles of $\alpha$ as $z_{1}-z_{j}, j=2, \ldots, l$, counting multiplicity.

Assume first that $\alpha$ has a pole of order 4 at one of $z_{1}-z_{j}$ 's, which without loss of generality we can assume to be $z_{1}-z_{2}$. Then set

$$
\gamma=\left(z_{1}-z_{2}\right)^{-4} \rho_{12}^{(-4)} \alpha .
$$

Obviously, $\gamma \in R^{l}$ and satisfies (i). Since $\gamma$ does not have poles at $z_{1}-z_{j}, z_{2}-z_{j}$ for $j \geqslant 3$, and (2.12) implies that $\operatorname{ord}_{i j} \gamma \geqslant \operatorname{ord}_{i j} \alpha$ for all $3 \leqslant i<j \leqslant l, \gamma$ satisfies (ii) as well. Therefore, the function $\alpha^{\prime}=\alpha-\gamma \in R^{l}$ has fewer multiple poles at $z_{1}-z_{j}$. By induction, $\alpha^{\prime}=\sum_{m} \alpha_{m}^{\prime}$ for $\alpha_{m} \in R^{l}$ satisfying (i) and (ii), and hence $\alpha=\gamma+\sum_{m} \alpha_{m}^{\prime}$.

Now assume that $\alpha$ has a double pole at some $z_{1}-z_{j}$, which is again can be taken $z_{1}-z_{2}$. Then set $\beta\left(z_{2}, \ldots, z_{l}\right)=\rho_{i j}^{(-2)} \alpha \in R^{l-1}$. By induction, we have $\beta=\sum_{m} \beta_{m}$, where the functions $\beta_{m} \in R^{l-1}$ satisfy conditions (i) and (ii) for $z_{i}=z_{2}$. For each $\beta_{m}$, we need to consider two cases, that correspond to the dichotomy of the condition (i):

Case 1. The function $\beta_{m}$ has a pole of order 4 at some $z_{2}-z_{j}$ for $j=3, \ldots, l$. Without loss of generality, we can assume that $j=3$. Then $\beta_{m}=\left(z_{2}-z_{3}\right)^{(-4)} \beta_{m}^{\prime}$ for some $\beta_{m}^{\prime} \in R^{l-3}$, and we set

$$
\gamma_{m}=\left(z_{1}-z_{2}\right)^{-2}\left(z_{1}-z_{3}\right)^{-2}\left(z_{2}-z_{3}\right)^{-2} \beta_{m}^{\prime} .
$$

Case 2. The function $\beta_{m}$ has poles of orders at most 2 at all $z_{2}-z_{j}$ for $j=3, \ldots, l$. Without loss of generality, we can assume that $\operatorname{ord}_{2 j} \beta_{m} \geqslant 1$ for $j \geqslant 5$. The we set

$$
\gamma_{m}=\left(z_{1}-z_{2}\right)^{-2}\left(z_{1}-z_{3}\right)^{-1}\left(z_{1}-z_{4}\right)^{-1}\left(z_{2}-z_{3}\right)\left(z_{2}-z_{4}\right) \beta_{m} .
$$


We need to show that in both cases the function $\gamma_{m}$ is admissible, satisfies conditions (i) and (ii) and $\rho_{12}^{(-2)} \gamma_{m}=\beta_{m}$. Indeed, assume that these properties of $\gamma_{m}$ are established. Then set $\alpha^{\prime}=\alpha-\sum_{m} \gamma_{m}$. Since $\gamma_{m}$ satisfies (ii), and $\rho_{12}^{(-2)} \alpha^{\prime}=0$, the function $\alpha^{\prime}$ will have less multiple poles in $z_{1}-z_{j}$ than $\alpha$, therefore by induction, $\alpha^{\prime}=\sum_{m} \alpha_{m}^{\prime}$ for $\alpha_{m}^{\prime} \in R^{l}$ satisfying conditions (i) and (ii), and we take expansion $\alpha=\sum_{m} \gamma_{m}+\sum_{m} \alpha_{m}^{\prime}$.

Note that the conditions (i) and $\rho_{12}^{(-2)} \gamma_{m}=\beta_{m}$ are obvious in both cases.

Case 1 is similar to the case when $\operatorname{ord}_{12} \alpha=-4$. We see that $\gamma_{m}$ is admissible by the definition. Since $\operatorname{ord}_{23} \beta=-4$ and $\operatorname{ord}_{12} \alpha=-2$, we must have $\operatorname{ord}_{13} \alpha \leqslant-2$, which together with (2.12) establishes the property (ii) for $\gamma_{m}$.

So assume we are in Case 2. Condition (ii) follows from (2.12) and the fact that the only multiple pole of $\gamma_{m}$ at $z_{1}-z_{j}$ and $z_{2}-z_{j}$ is at $z_{1}-z_{2}$. We are left to show that $\gamma_{m}$ is admissible.

As it was mentioned above, we can assume that $\beta_{m}=\beta_{m 1} \beta_{m 2} \cdots$ is a product of indecomposable admissible functions $\beta_{m t} \in R^{l_{t}}, \quad \sum_{t} l_{t}=l-1$. Suppose $\beta_{l 1}$ depends on $z_{2}$. Then $l_{1}>2$, since $\operatorname{ord}_{23} \beta_{m}=-2$. Since our choice of $z_{3}$ and $z_{4}$ was based only on the condition that $\operatorname{ord}_{2 j} \beta_{m} \geqslant-1$ for $j \neq 3,4$, we can assume that $\beta_{m 1}$ depends on $z_{3}$ and $z_{4}$. Therefore, in order to prove that $\gamma_{m}$ is admissible, it is enough to show that if $\beta_{m}$ is indecomposable admissible, then so is $\gamma_{m}$.

So assume that $\beta_{m} \in R_{0}^{l-1}$. Applying $\Delta^{*}$ to $\gamma_{m}$ and using (2.14) and the fact that $\beta_{m}$ is regular, we see that $\gamma_{m}$ is regular as well. So we are left to verify that for every partition $\{1, \ldots, l\}=I \sqcup J$ we have $\left(\gamma_{m}\right)_{n}=0$ for $n \leqslant 1$.

Using Corollary 3.1, we can assume without loss of generality that $1 \in I$; otherwise we could swap $I$ and $J$. Let

$$
\left(\beta_{m}\right)_{n}=\sum_{j}\left(\beta_{m}\right)_{-n, j}^{\prime}\left(\beta_{m}\right)_{n, j}^{\prime \prime}
$$

be the component of $\beta_{m}$ corresponding to partition $\{2, \ldots, l\}=(I \backslash\{1\}) \sqcup J$. Since $\beta_{m} \in R_{0}^{l-1}$, we have $\left(\beta_{m}\right)_{n}=0$ for $n \leqslant 1$. We can expand the factor

$$
\varkappa=\left(z_{1}-z_{2}\right)^{-2}\left(z_{1}-z_{3}\right)^{-1}\left(z_{1}-z_{4}\right)^{-1}\left(z_{2}-z_{3}\right)\left(z_{2}-z_{4}\right)
$$

in (3.8) as $\varkappa=\sum_{s} \varkappa_{s}^{\prime} \varkappa_{s}^{\prime \prime}$, where $\varkappa_{s}^{\prime}$ depends on the variables $\left\{z_{i} \mid i \in I \cap\{1,2,3,4\}\right\}$ and $\varkappa_{s}^{\prime \prime}$ depends on the variables $\left\{z_{i} \mid i \in J \cap\{1,2,3,4\}\right\}$, so that $\operatorname{deg} \varkappa_{s}^{\prime \prime} \geqslant 0$. We use here that $z_{1}$ appears in $\varkappa_{s}^{\prime}$. Then the decomposition (3.1) for $\gamma_{m}$ becomes

$$
\sum_{n, j, s}\left(\varkappa_{s}^{\prime}\left(\beta_{m}\right)_{-n, j}^{\prime}\right)\left(\varkappa_{s}^{\prime \prime}\left(\beta_{m}\right)_{n, j}^{\prime \prime}\right)
$$

therefore, $\left(\gamma_{m}\right)_{n}=0$ for $n \leqslant 1$.

The proof of Proposition 3.4 is very similar to the proof of Lemma 3.1.

Proof of Proposition 3.4. We use induction on the number of non-zero functions among $\left\{\alpha_{i j}^{(k)} \mid k=-2,-4,1 \leqslant i<j \leqslant l\right\}$. If all of them are 0 , then take $\alpha=0$.

Assume first that some $\alpha_{i j}^{(-4)} \neq 0$. To simplify notations, we can take $\alpha_{12}^{(-4)} \neq 0$. Then set

$$
\gamma=\left(z_{1}-z_{2}\right)^{-4} \alpha_{12}^{(-4)} \in R^{l}
$$

As before, we see that $\gamma$ does not have poles at $\left(z_{1}-z_{j}\right),\left(z_{2}-z_{j}\right)$ for $j \geqslant 3$ and $\operatorname{ord}_{i j} \gamma \geqslant \operatorname{ord}_{i j} \alpha$ for $3 \leqslant i<j \leqslant l$, therefore the collection $\left\{\alpha_{i j}^{(k)}-\rho_{i j}^{(k)} \gamma\right\}$ has fewer non-zero terms. This 
collection satisfies the condition (3.6) because of the property $(2.12)$ of the coefficients $\rho_{i j}^{(k)} \gamma$. By induction, there is a function $\alpha^{\prime}$, such that $\rho_{i j}^{(k)} \alpha^{\prime}=\alpha_{i j}^{(k)}-\rho_{i j}^{(k)} \gamma$, and we can take $\alpha=\alpha^{\prime}+\gamma$.

Now assume that $\alpha_{i j}^{(-4)}=0$, but $\beta=\alpha_{i j}^{(-2)} \neq 0$, for some $1 \leqslant i<j \leqslant l$, which again can be assumed to be 1 and 2 . Then by Lemma 3.1, we can write $\beta\left(z_{2}, \ldots, z_{l}\right)=\sum_{m} \beta_{m}$ for some functions $\beta_{m} \in R^{l-1}$ satisfying the conditions (i) and (ii) of Lemma 3.1 for $z_{i}=z_{2}$. Exactly as in the proof of Lemma 3.1, without loss of generality we can consider two cases for each $\beta_{m}$ : when $\beta_{m}$ has a pole of order 4 at $z_{2}-z_{3}$ and when $\beta_{m}$ might have double poles at $z_{2}-z_{3}$ and $z_{2}-z_{4}$ but at most simple poles at $z_{2}-z_{j}$ for $j \geqslant 5$. In each of these cases define the function $\gamma_{m}$ by the formulas (3.7) and (3.8) respectively. As before, we see each $\gamma_{m}$ is admissible, satisfies the property (ii) of Lemma 3.1 and $\rho_{12}^{(-2)} \gamma_{m}=\beta_{m}$. Therefore, setting $\gamma=\sum_{m} \gamma_{m}$ as before, we see that the collection $\left\{\alpha_{i j}^{(k)}-\rho_{i j}^{(k)} \gamma\right\}$ has fewer non-zero terms and satisfies (3.6), so we finish proof of the Proposition using induction as above.

\section{The coalgebras of correlation functions}

\subsection{Spaces of correlation functions}

Let $V=\bigoplus_{d} V_{d}$ be a vertex algebra with $s l_{2}$ structure. Assume that it has a set of homogeneous generators $\mathcal{G} \subset V$ such that $D^{*} \mathcal{G}=0$.

Remark 4.1. The results in this section could be extended to the case when the generators $\mathcal{G}$ are not necessarily minimal, but we do not need this generalization here.

Set $T(\mathcal{G})=\left\{a_{1} \otimes \cdots \otimes a_{l} \in V^{\otimes l} \mid a_{i} \in \mathcal{G}\right\}$. For any $\boldsymbol{a}=a_{1} \otimes \cdots \otimes a_{l} \in T(\mathcal{G})$, consider the space

$$
V^{\boldsymbol{a}}=\operatorname{Span}_{\mathbb{k}}\left\{a_{1}\left(n_{1}\right) \cdots a_{l}\left(n_{l}\right) \mathbb{1} \mid n_{i} \in \mathbb{Z}\right\} \subset V .
$$

Denote $V_{d}^{\boldsymbol{a}}=V^{\boldsymbol{a}} \cap V_{d}$. The commutativity property of correlation functions (see Section 2.2) implies that for any permutation $\sigma \in \Sigma_{l}$ and a scalar $k \in \mathbb{k}$ we have $V^{\sigma \boldsymbol{a}}=V^{k \boldsymbol{a}}=V^{\boldsymbol{a}}$.

For a tensor $\boldsymbol{a}=a_{1} \otimes \cdots \otimes a_{l} \in T(\mathcal{G})$ and a subsequence $I=\left\{i_{1}, i_{2}, \ldots\right\} \subset\{1, \ldots, l\}$ define $\boldsymbol{a}(I)=a_{i_{1}} \otimes a_{i_{2}} \otimes \cdots \in T(\mathcal{G})$.

As it was explained in Section 2.2, to any linear functional $f: V_{d}^{\boldsymbol{a}} \rightarrow \mathbb{k}$ we can correspond a correlation function $\alpha_{f} \in \Phi^{l}$ of degree $d-\sum_{i} \operatorname{deg} a_{i}$, such that $\operatorname{ord}_{i j} \alpha \geqslant-\operatorname{loc}\left(a_{i}, a_{j}\right)$. Let

$$
\Omega^{a}=\bigoplus_{d} \Omega_{d}^{a}, \quad \Omega_{d}^{a}=\left\{\alpha_{f} \mid f: V_{d}^{a} \rightarrow \mathbb{k}\right\} \subset \Phi_{d-\sum \operatorname{deg} a_{i}}^{l}
$$

be the space of all such correlation functions, so that $\left(V_{d}^{\boldsymbol{a}}\right)^{*} \cong \Omega_{d}^{\boldsymbol{a}}$.

Definition 4.1. We will call the space

$$
\Omega=\Omega(V)=\bigoplus_{\boldsymbol{a} \in T(\mathcal{G})} \Omega^{\boldsymbol{a}}
$$

the vertex coalgebra of correlation functions of a vertex algebra $V$.

Note that $\Omega(V)$ depends on the choice of generators $\mathcal{G}$, though we supress this dependence in the notation $\Omega(V)$. Also note that while each homogeneous component $\Omega^{\boldsymbol{a}} \subset \Phi^{|\boldsymbol{a}|}$ consists of rational functions, the whole space $\Omega(V)$ is not a subspace of $\Phi$.

The coalgebra structure on $\Omega(V)$, similar to the one defined in [13], is manifested in the following properties, which easily follow from the properties of vertex algebras (see Section 2): 
S0. $\Omega^{1}=\mathbb{k}, \Omega^{a}=\mathbb{k}[z]$ for every $a \in \mathcal{G}$, and for $\boldsymbol{a}=a_{1} \otimes \cdots \otimes a_{l} \in T(\mathcal{G}), l \geqslant 2$.

$\Omega 1$. $\operatorname{ord}_{i j} \alpha \geqslant-\operatorname{loc}\left(a_{i}, a_{j}\right)$ for any $\alpha \in \Omega^{a}$.

ת2. $\Omega^{a}=\sigma \Omega^{\sigma a}$ for any permutation $\sigma \in \Sigma_{l}$.

$\Omega 3$. The space $\Omega^{\boldsymbol{a}}$ is closed under the operators $\Delta=\sum_{i} \partial_{z_{i}}$ and $\Delta^{*}=\sum_{i}\left(z_{i}^{2} \partial_{z_{i}}+2\left(\operatorname{deg} a_{i}\right) z_{i}\right)$.

$\Omega 4$. Set $\boldsymbol{b}=a_{2} \otimes \cdots \otimes a_{l} \in T(\mathcal{G})$. Then any function $\alpha \in \Omega^{\boldsymbol{a}}$ can be expanded at $z_{1}=\infty$ into a series

$$
\alpha\left(z_{1}, \ldots, z_{l}\right)=\sum_{n \geqslant n_{0}} z_{1}^{-n-1} \alpha_{n}\left(z_{2}, \ldots z_{l}\right)
$$

where $\alpha_{n} \in \Omega^{b}$.

The action of $\Sigma_{l}$ on $\Omega^{\boldsymbol{a}}$ in $(\Omega 2)$ is defined by $(\sigma \alpha)\left(z_{1} \ldots, z_{l}\right)=\alpha\left(z_{\sigma(1)}, \ldots, z_{\sigma(l)}\right)$, so that $(\Omega 2)$ is just the commutativity property of Section 2.2. It implies that the space $\Omega^{\boldsymbol{a}}$ for $\boldsymbol{a}=$ $a_{1} \otimes \cdots \otimes a_{l} \in T(\mathcal{G})$ is symmetric under the group $\Gamma_{\boldsymbol{a}} \subset \Sigma_{l}$ generated by all transpositions $(i j)$ whenever $a_{i}=a_{j}$.

Note that in order to get the expansion (3.1) of a function $\alpha \in \Omega^{\boldsymbol{a}}$, we need to apply a suitable permutation to the variables $z_{1}, \ldots, z_{n}$, and then iterate the expansion (4.1) several times. Combining this observation with the property $(\Omega 2)$, we see that $(\Omega 4)$ can be reformulated as follows:

$\Omega 4^{\prime}$. For a partition $\{1, \ldots, l\}=I \sqcup J$, denote $\boldsymbol{a}^{\prime \prime}=\boldsymbol{a}(J)$. Then the component of degree $n$ of a function $\alpha\left(z_{1}, \ldots, z_{l}\right) \in \Omega^{a}$ of degree $d-\sum_{i} \operatorname{deg} a_{i}$ can be written as $(\alpha)_{s}=\sum_{j} \alpha_{d-n, j}^{\prime} \alpha_{n, j}^{\prime \prime}$ so that $\alpha_{n, j}^{\prime \prime} \in \Omega^{a^{\prime \prime}}$.

\subsection{Universal vertex algebras}

Now we want to present a converse construction: given a space of functions $\Omega$, satisfying the conditions $(\Omega 0)-(\Omega 4)$, we will construct a vertex algebra $V=V(\Omega)$, such that $\Omega=\Omega(V)$.

Let $\mathcal{G}$ be a set. For any $a \in \mathcal{G}$ fix its degree $\operatorname{deg} a \in \mathbb{Z}$, and for any pair $a, b \in \mathcal{G}$ fix a number $\operatorname{loc}(a, b) \in \mathbb{Z}$.

Theorem 4.1. Let $\Omega=\bigoplus_{\boldsymbol{a} \in T(\mathcal{G})} \Omega^{\boldsymbol{a}}$ be a graded space constructed from rational functions as above, satisfying conditions $(\Omega 0)-(\Omega 4)$. Then there exists a vertex algebra

$$
V=V(\Omega)=\bigoplus_{\lambda \in \mathbb{Z}_{+}[\mathcal{G}]} V^{\lambda}
$$

generated by $\mathcal{G}$ so that $a \in V_{\operatorname{deg} a}, D^{*} a=0$ for any $a \in \mathcal{G}$, such that $\Omega=\Omega(V)$ is the vertex coalgebra of correlation functions of $V$ (see Definition 4.1).

Proof. Let $\Omega_{d}^{a} \subset \Omega^{a}$ be the subspace of functions of degree $d-\sum_{i} \operatorname{deg} a_{i}$. The condition $(\Omega 1)$ implies that $\Omega^{a}=\bigoplus_{d} \Omega_{d}^{a}$ so that $\Omega_{d}^{a}=0$ when $d \ll 0$ and $\operatorname{dim} \Omega_{d}^{a}<\infty$.

For each $\boldsymbol{a} \in T(\mathcal{G})$ set $V^{\boldsymbol{a}}=\left(\Omega^{\boldsymbol{a}}\right)^{\prime}$ to be the graded dual space of $\Omega^{\boldsymbol{a}}$. We define degree on $V^{\boldsymbol{a}}$ by setting $\operatorname{deg} v=d+\sum_{i} \operatorname{deg} a_{i}$ for $v: \Omega_{d}^{\boldsymbol{a}} \rightarrow \mathbb{k}$. For a permutation $\sigma \in \Sigma_{l}$ we identify $V^{\boldsymbol{a}}$ with $V^{\sigma a}$ using $(\Omega 2)$. In this way for every $\lambda=a_{1}+\cdots+a_{l} \in \mathbb{Z}_{+}[\mathcal{G}]$ we obtain a space $V^{\lambda}=V^{a_{1} \otimes \cdots \otimes a_{l}}$, and set $V=V(\Omega)=\bigoplus_{\lambda \in \mathbb{Z}_{+}[\mathcal{G}]} V^{\lambda}$.

For every $\lambda=a_{1}+\cdots+a_{l} \in \mathbb{Z}_{+}[\mathcal{G}]$ choose a basis $\mathcal{B}_{d}^{\lambda}$ of $V_{d}^{\lambda}$. Set $\mathcal{B}^{\lambda}=\bigcup_{d} \mathcal{B}_{d}^{\lambda}$. Let $\left\{\alpha_{u} \mid u \in\right.$ $\left.\mathcal{B}_{d}^{\lambda}\right\}$ be the dual basis of $\Omega_{d}^{\boldsymbol{a}}$, where $\boldsymbol{a}=a_{1} \otimes \cdots \otimes a_{l} \in T(\mathcal{G})$, so that $\operatorname{deg} \alpha_{u}=\operatorname{deg} u-\sum_{i} \operatorname{deg} a_{i}$. For a permutation $\sigma \in \Sigma_{l}$ the set $\left\{\sigma \alpha_{u} \mid u \in \mathcal{B}_{d}^{\lambda}\right\}$ is the basis of $\Omega_{d}^{\sigma a}$, dual to $\mathcal{B}_{d}^{\lambda}$. 
We choose these bases so that $\mathcal{B}^{0}=\{\mathbb{1}\}$ and $\alpha_{\mathbb{1}}=1 \in \Omega^{0}=\mathbb{k}$. Also, for a generator $a \in \mathcal{G}$ of degree $d$ we have $\operatorname{dim} V_{d}^{a}=1$, since $V_{d}^{a}=\left(\Omega_{0}^{a}\right)^{*}$ and $\Omega_{0}^{a}=\mathbb{k}$ due to $(\Omega 0)$. We can identify the only element of $\mathcal{B}_{d}^{a}$ with $a$ so that $\alpha_{a}=1 \in \Omega_{0}^{a}$.

We define the operators $D: V^{\lambda} \rightarrow V^{\lambda}$ and $D^{*}: V^{\lambda} \rightarrow V^{\lambda}$ as the duals to $\Delta: \Omega^{a} \rightarrow \Omega^{a}$ and $\Delta^{*}\left(2 \operatorname{deg} a_{1}, \ldots, 2 \operatorname{deg} a_{l}\right): \Omega^{\boldsymbol{a}} \rightarrow \Omega^{\boldsymbol{a}}$ respectively (see Section 2.3). Since $\Delta^{*}(2 \operatorname{deg} a) \Omega^{a} \subset z \operatorname{k}[z]$, we have $D^{*} a=0$ for every $a \in \mathcal{G}$.

Now we are going to define vertex algebra structure $Y: V \rightarrow \operatorname{Hom}(V, V((z)))$ so that for any $\lambda=a_{1}+\cdots+a_{l} \in \mathbb{Z}_{+}[\mathcal{G}]$ we have

$$
\begin{aligned}
& Y\left(a_{1}, z_{1}\right) \cdots Y\left(a_{l}, z_{l}\right) \mathbb{1}=\sum_{u \in \mathcal{B}^{\lambda}} \alpha_{u}\left(z_{1}, \ldots, z_{l}\right) u, \\
& Y\left(a_{1}, z_{1}+z\right) \cdots Y\left(a_{l}, z_{l}+z\right) w=Y\left(Y\left(a_{1}, z_{1}\right) \cdots Y\left(a_{l}, z_{l}\right) \mathbb{1}, z\right) w .
\end{aligned}
$$

These identities are to be understood in the following sense. The left-hand side of (4.2) converges to the $V$-valued rational function on the right-hand side in the region $\left|z_{1}\right|>\left|z_{2}\right|>\cdots>\left|z_{l}\right|$. The left and right-hand sides of (4.3) converge to the same $V$-valued rational function in the regions $\left|z_{1}+z\right|>\left|z_{2}+z\right|>\cdots>\left|z_{l}+z\right|$ and $|z|>\left|z_{1}\right|>\left|z_{2}\right|>\cdots>\left|z_{l}\right|$ respectively.

Take, as before, $\lambda=a_{1}+\cdots+a_{l} \in \mathbb{Z}_{+}[\mathcal{G}]$ and $\boldsymbol{a}=a_{1} \otimes \cdots \otimes a_{l} \in T(\mathcal{G})$. Let $a \in \mathcal{G}$ be a generator of degree $d$. First we define the action of $Y(a, z)$ on $V^{\lambda}$.

For any $v \in \mathcal{B}^{a+\lambda}$ expand the corresponding basic function $\alpha_{v}$ as in (4.1):

$$
\alpha_{v}\left(z, z_{1}, \ldots, z_{l}\right)=\sum_{n} z^{-n-1} \alpha_{n}\left(z_{1}, \ldots, z_{l}\right), \quad \alpha_{n} \in \Omega^{a} .
$$

Expand $\alpha_{n}$ in the basis of $\Omega^{a}$, and get

$$
\alpha_{v}=\sum_{u \in \mathcal{B}^{\lambda}} c_{u v} z^{\operatorname{deg} v-\operatorname{deg} u-d} \alpha_{u}
$$

for some $c_{u v} \in \mathbb{k}$. Now set

$$
Y(a, z) u=\sum_{v \in \mathcal{B}^{a+\lambda}} c_{u v} z^{\operatorname{deg} v-\operatorname{deg} u-d} v
$$

for any $u \in \mathcal{B}^{\lambda}$, and extend it by linearity to the whole $V^{\lambda}$.

For example, take $\lambda=0$. Assume that $\mathcal{B}^{a}=\left\{a, D a, D^{2} a, \ldots\right\}$, then $\alpha_{D^{m} a}=\frac{1}{m !} z^{m} \in \Omega_{m}^{a}$, and therefore

$$
Y(a, z) \mathbb{1}=\sum_{m \geqslant 0} \frac{1}{m !} z^{m} D^{m} a,
$$

which agrees with the identity (2.2).

It is easy to check that (4.2) is satisfied: Indeed, we have checked that it holds for $\lambda=0$; assuming that it holds for $\lambda=a_{1}+\cdots+a_{l}$, we compute, using (4.4),

$$
\begin{aligned}
Y(a, z) Y\left(a_{1}, z_{1}\right) \cdots Y\left(a_{l}, z_{l}\right) \mathbb{1} & =\sum_{u \in \mathcal{B}^{\lambda}} Y(a, z) u \alpha_{u}\left(z_{1}, \ldots, z_{l}\right) \\
& =\sum_{u \in \mathcal{B}^{\lambda}, v \in \mathcal{B}^{a+\lambda}} c_{u v} z^{\operatorname{deg} v-\operatorname{deg} u-d} \alpha_{u}\left(z_{1}, \ldots, z_{l}\right) v \\
& =\sum_{v \in \mathcal{B}^{a+\lambda}} \alpha_{v}\left(z, z_{1}, \ldots, z_{l}\right) v .
\end{aligned}
$$

In order to show that the correspondence $\mathcal{G} \ni a \mapsto Y(a, z) \in \operatorname{Hom}(V, V((z)))$ can be extended to a map $Y: V \rightarrow \operatorname{Hom}(V, V((z)))$, we need to introduce another property of $\Omega$ : 
$\Omega 5$. If $\boldsymbol{a}=\boldsymbol{a}^{\prime} \otimes \boldsymbol{a}^{\prime \prime}$ for $\boldsymbol{a}^{\prime}, \boldsymbol{a}^{\prime \prime} \in T(\mathcal{G}),\left|\boldsymbol{a}^{\prime}\right|=k,\left|\boldsymbol{a}^{\prime \prime}\right|=l-k$, then any function $\alpha \in \Omega^{\boldsymbol{a}}$ has an expansion

$$
\alpha\left(z_{1}+z, \ldots, z_{k}+z, z_{k+1}, \ldots, z_{l}\right)=\sum_{n \geqslant n_{0}} z^{-n-1} \sum_{i} \alpha_{n i}^{\prime}\left(z_{1}, \ldots, z_{k}\right) \alpha_{n i}^{\prime \prime}\left(z_{k+1}, \ldots, z_{l}\right)
$$

at $z=\infty$, where $\alpha_{n i}^{\prime} \in \Omega^{a^{\prime}}$ and $\alpha_{n i}^{\prime \prime} \in \Omega^{a^{\prime \prime}}$. The second sum here is finite.

Note that if $k=l$, then the expansion (4.5) just the usual Taylor formula

$$
\alpha\left(z_{1}+z, \ldots, z_{l}+z\right)=\exp (\Delta z) \alpha\left(z_{1}, \ldots, z_{l}\right),
$$

since the left-hand side is polynomial in $z$.

Lemma 4.1. Let $\Omega=\bigoplus_{\boldsymbol{a} \in T(\mathcal{G})} \Omega^{\boldsymbol{a}}$ be a homogeneous space of rational functions, satisfying the conditions $(\Omega 0)-(\Omega 4)$ of Section 4.1 . Then it also satisfies $(\Omega 5)$.

Before proving this lemma, let us show how condition $(\Omega 5)$ helps to construct the vertex algebra structure on $V$, and hence proving Theorem 4.1. Take two weights $\lambda=a_{1}+\cdots+a_{l}$, $\mu=b_{1}+\cdots+b_{k} \in \mathbb{Z}_{+}[\mathcal{G}]$, and define the tensors $\boldsymbol{a}=a_{1} \otimes \cdots \otimes a_{l}, \boldsymbol{b}=b_{1} \otimes \cdots \otimes b_{k} \in T(\mathcal{G})$. We are going to define the action of $Y\left(V^{\lambda}, z\right)$ on $V^{\mu}$ and then by linearity extend $Y$ to the whole $V$.

In analogy with deriving (4.4), we obtain from (4.5) that every basic function $\alpha_{v} \in \Omega^{\boldsymbol{a} \otimes \boldsymbol{b}}$ has expansion

$$
\begin{aligned}
\alpha_{v}\left(z_{1}\right. & \left.+z, \ldots, z_{l}+z, y_{1}, \ldots, y_{k}\right) \\
& =\sum_{u \in \mathcal{B}^{\lambda}, w \in \mathcal{B}^{\mu}} c_{u, w}^{v} z^{\operatorname{deg} v-\operatorname{deg} u-\operatorname{deg} w} \alpha_{u}\left(z_{1}, \ldots z_{l}\right) \alpha_{w}\left(y_{1}, \ldots, y_{k}\right),
\end{aligned}
$$

for some $c_{u, w}^{v} \in \mathbb{k}$. Now we set for $u \in \mathcal{B}^{\lambda}$ and $w \in \mathcal{B}^{\mu}$

$$
Y(u, z) w=\sum_{v \in \mathcal{B}^{\lambda+\mu}} c_{u, w}^{v} z^{\operatorname{deg} v-\operatorname{deg} u-\operatorname{deg} w} v .
$$

To check (4.3), sum (4.6) over all $v \in \mathcal{B}^{\lambda+\mu}$. By (4.2), the left-hand is

$$
Y\left(a_{1}, z_{1}+z\right) \cdots Y\left(a_{l}, z_{l}+z\right) Y\left(b_{1}, y_{1}\right) \cdots Y\left(b_{k}, y_{k}\right) \mathbb{1}
$$

whereas the right-hand side is, using the definition of $Y(u, z) w$ and (4.2),

$$
\begin{aligned}
& \sum_{u \in \mathcal{B}^{\lambda}, w \in \mathcal{B}^{\mu}} Y(u, z) w \alpha_{u}\left(z_{1}, \ldots, z_{l}\right) \alpha_{w}\left(y_{1}, \ldots, y_{k}\right) \\
& \quad=Y\left(Y\left(a_{1}, z_{1}\right) \cdots Y\left(a_{l}, z_{l}\right) \mathbb{1}, z\right) Y\left(b_{1}, y_{1}\right) \cdots Y\left(b_{k}, y_{k}\right) \mathbb{1} .
\end{aligned}
$$

It remains to be seen that the map $Y: V \rightarrow \operatorname{Hom}(V, V((z)))$ defines a structure of vertex algebra on $V$. By the construction, $Y$ satisfies (2.2) and (2.3), and the identity (4.2) guarantees that the correlation functions for $Y$ satisfy the rationality and commutativity conditions, which, as it was observed in Section 2.2, are enough for $V$ to be a vertex algebra.

Note also that $Y$ does not depend on the choice of the bases $\mathcal{B}^{\lambda}$, since it depends only on the tensors $\sum_{u \in \mathcal{B}_{d}^{\lambda}} u \otimes \alpha_{u} \in V_{d}^{\lambda} \otimes \Omega^{a}$.

It is easy to see that the vertex algebra $V=V(\Omega)$ has the following universality property:

Proposition 4.1. Let $U$ be a vertex algebra, generated by the set $\mathcal{G} \subset U$, such that the coalgebra of generating functions $\Omega(U)$ (given by Definition 4.1) is a subspace of $\Omega$. Then there is a unique vertex algebra homomorphism $V \rightarrow U$ that fixes $\mathcal{G}$.

Remark 4.2. In Section 4.1 we have constructed a vertex coalgebra $\Omega=\Omega(V)$ of correlation functions of a vertex algebra $V$ (see Definition 4.1). If we apply the construction of Theorem 4.1 to this $\Omega$, we get $V(\Omega)=\bigoplus_{\lambda \in \mathbb{Z}_{+}[\mathcal{G}]} V^{\lambda}$, which is the graded deformation algebra (a.k.a. the Rees algebra) of $V$. 


\subsection{Example: Free vertex algebra}

Clearly the conditions $(\Omega 0)-(\Omega 4)$ are satisfied for

$$
\Omega^{\boldsymbol{a}}=\left\{\alpha \in \Phi^{l} \mid \operatorname{ord}_{i j} \alpha \geqslant-\operatorname{loc}\left(a_{i}, a_{j}\right) \forall 1 \leqslant i<j \leqslant l\right\}^{\Gamma_{a}} .
$$

By Proposition 4.1, the resulting vertex algebra $F=F_{\text {loc }}(\mathcal{G})=V(\Omega)$, given by Theorem 4.1, has the following universal property: any vertex algebra $U$ generated by the set $\mathcal{G}$ such that the locality of any $a, b \in \mathcal{G}$ is at most $\operatorname{loc}(a, b)$, is a homomorphic image of $F$. Such a vertex algebra $F$ is called a free vertex algebra. It was constructed in $[22,23]$ using different methods.

\subsection{Proof of Lemma 4.1}

Take some $\alpha \in \Omega_{d}^{a}$. As it is the case with any rational function with poles at $z_{i}-z_{j}$ only, $\alpha$ has an expansion (4.5). We just have to show that $\alpha_{n i}^{\prime} \in \Omega^{\boldsymbol{a}^{\prime}}$ and $\alpha_{n i}^{\prime \prime} \in \Omega^{\boldsymbol{a}^{\prime \prime}}$.

First we show that any $\alpha_{n i}^{\prime \prime}$ in (4.5) belongs to $\Omega^{\boldsymbol{a}^{\prime \prime}}$. Let $\alpha^{\prime \prime}\left(z_{k+1}, \ldots, z_{l}\right)$ be the coefficient of some monomial $z^{-n-1} z_{1}^{-n_{1}-1} \cdots z_{k}^{-n_{k}-1}$ in (4.5). Clearly, it is enough to show that this $\alpha^{\prime \prime} \in \Omega^{a^{\prime \prime}}$. The idea is that $\alpha^{\prime \prime}$ is a finite linear combination of the coefficients of $z_{1}^{-m_{1}-1} \cdots z_{k}^{-m_{k}-1}$ in the expansion of $\alpha$ in the domain $\left|z_{1}\right|>\cdots>\left|z_{l}\right|$, which are in $\Omega^{a^{\prime \prime}}$ by $\left(\Omega 4^{\prime}\right)$. While this can be shown by some manipulations with rational functions, we will use some vertex algebra considerations.

Namely, we are going to use the free vertex algebra $F=F_{\text {loc }}(\mathcal{G})$, discussed in Section 4.3. Since every function $\alpha \in \Phi^{l}$ satisfying $(\Omega 1)$ is a correlation function on $F$, there is a linear functional $f: F_{d}^{a} \rightarrow \mathbb{k}$ such that $\alpha=\alpha_{f}$ is the correlation function of $f$, given by (2.9). By the associativity property (see Section 2.2), we have that $\alpha^{\prime \prime}=\alpha_{f^{\prime \prime}}$ is the correlation function of the functional $f^{\prime \prime}: F_{d^{\prime \prime}}^{\boldsymbol{a}^{\prime \prime}} \rightarrow \mathbb{k}$, given by $v \mapsto f\left(\left(a_{1}\left(n_{1}\right) \cdots a_{k}\left(n_{k}\right) \mathbb{1}\right)(n) v\right)$, where $d^{\prime \prime}=d-$ $\operatorname{deg}\left(a_{1}\left(n_{1}\right) \cdots a_{k}\left(n_{k}\right) \mathbb{1}\right)(n)$. Using the identity $(2.5)$, we see that $\left(a_{1}\left(n_{1}\right) \cdots a_{k}\left(n_{k}\right) \mathbb{1}\right)(n)$ as an operator $F_{d^{\prime \prime}}^{\boldsymbol{a}^{\prime \prime}} \rightarrow F_{d}^{\boldsymbol{a}}$ can be represented as a linear combination of words $u=a_{i_{1}}\left(m_{1}\right) \cdots a_{i_{k}}\left(m_{k}\right) \in$ $U(F)$ for some $m_{i} \in \mathbb{Z}$ and a permutation $\sigma=\left(i_{1}, \ldots, i_{k}\right) \in \Sigma_{k}$. But the correlation function of

the functional $v \mapsto f(u v)$ for such $u$ is the coefficient of $z_{i_{1}}^{-m_{1}-1} \cdots z_{i_{k}}^{-m_{k}-1}$ in the expansion of $\sigma \alpha$ in the domain $\left|z_{i_{1}}\right|>\cdots>\left|z_{i_{k}}\right|>\left|z_{k+1}\right|>\cdots>\left|z_{l}\right|$, and therefore belongs to $\Omega^{\boldsymbol{a}^{\prime \prime}}$ by $\left(\Omega 4^{\prime}\right)$.

Remark 4.3. Actually, one can show that it suffices to use only words $u$ with $\sigma=1$.

Now we prove that $\alpha_{n i}^{\prime} \in \Omega^{\boldsymbol{a}^{\prime}}$. Recall that $\sigma \Omega^{\boldsymbol{a}}=\Omega^{\sigma \boldsymbol{a}}$ for any permutation $\sigma \in \Sigma_{l}$. Apply the permutation that reverses the order of variables to (4.5), replace $z$ by $-z$, and then the above argument shows that the expansion of $\alpha\left(z_{1}, \ldots, z_{k}, z_{k+1}-z, \ldots, z_{l}-z\right)$ in $z$ at $\infty$ has form

$$
\alpha\left(z_{1}, \ldots, z_{k}, z_{k+1}-z, \ldots, z_{l}-z\right)=\sum_{n \geqslant n_{0}} z^{-n-1} \sum_{i} \widetilde{\alpha}_{n i}^{\prime}\left(z_{1}, \ldots, z_{k}\right) \widetilde{\alpha}_{n i}^{\prime \prime}\left(z_{k+1}, \ldots, z_{l}\right),
$$

where $\widetilde{\alpha}_{n i}^{\prime} \in \Omega^{\boldsymbol{a}^{\prime}}$. Now take another variable $w$ and consider a finite expansion

$$
\alpha\left(z_{1}+w, \ldots, z_{l}+w\right)=\sum_{j} w^{j} \alpha^{(j)}\left(z_{1}, \ldots, z_{l}\right)
$$

where $\alpha^{(j)}=\frac{1}{j !} \Delta^{j} \alpha \in \Omega^{a}$. Here we use $(\Omega 3)$ and the fact that $\Delta$ is locally nilpotent on $\Phi^{l}$. Then we have

$$
\begin{aligned}
\alpha\left(z_{1}\right. & \left.+w, \ldots, z_{k}+w, z_{k+1}+w-z, \ldots, z_{l}+w-z\right) \\
& =\sum_{j} w^{j} \alpha^{(j)}\left(z_{1}, \ldots, z_{k}, z_{k+1}-z, \ldots, z_{l}-z\right) \\
& =\sum_{j, n, i} w^{j} z^{-n-1}{\widetilde{\left(\alpha^{(j)}\right)}}_{n i}^{\prime}\left(z_{1}, \ldots, z_{k}\right) \widetilde{\left(\alpha^{(j)}\right)_{n i}^{\prime \prime}}\left(z_{k+1}, \ldots, z_{l}\right) .
\end{aligned}
$$


As we have seen, $\left(\alpha^{(j)}\right)_{n i}^{\prime} \in \Omega^{\boldsymbol{a}^{\prime}}$. Now substitute $w=z$ in the above, and get that $\alpha_{n i}^{\prime}$ is a finite

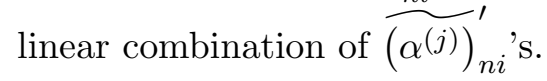

\subsection{Coalgebras of regular functions}

Suppose that we are in the setup of Section 4.2 , and that $\operatorname{deg} a \geqslant 0$ for any $a \in \mathcal{G}$.

Theorem 4.2. Let $\Omega_{0}=\bigoplus_{\boldsymbol{a} \in T(\mathcal{G})} \Omega_{0}^{\boldsymbol{a}}$, where $\Omega_{0}^{\boldsymbol{a}} \subset \Phi^{l}$, be a homogeneous space of functions of degree $-\sum_{i} \operatorname{deg} a_{i}$ for $\boldsymbol{a}=a_{1} \otimes \cdots \otimes a_{l} \in T(\mathcal{G})$. Assume that for any partition $\{1, \ldots, l\}=I \sqcup J$ the component decomposition (3.1) of a function $\alpha \in \Omega_{0}^{a}$ is

$$
\alpha=\sum_{n \geqslant 0}(\alpha)_{n}, \quad(\alpha)_{n}=\sum_{j} \alpha_{-n, j}^{\prime} \alpha_{n, j}^{\prime \prime}
$$

Assume also that

i. $\Omega_{0}^{1}=\mathrm{k}$;

ii. any $\alpha \in \Omega_{0}^{\boldsymbol{a}}$ is $\left(2 \operatorname{deg} a_{1}, \ldots, 2 \operatorname{deg} a_{l}\right)$-regular;

iii. $\operatorname{ord}_{i j} \alpha \geqslant-\operatorname{loc}\left(a_{i}, a_{j}\right)$ for every $1 \leqslant i<j \leqslant l$;

iv. $\sigma \Omega_{0}^{a}=\Omega_{0}^{\sigma a}$ for any permutation $\sigma \in \Sigma_{l}$;

v. for any $a_{1} \in \mathcal{G}$ there is a tensor $\boldsymbol{a}=a_{1} \otimes a_{2} \otimes \cdots \in T(\mathcal{G})$ such that $\Omega_{0}^{\boldsymbol{a}} \neq 0$;

vi. $\alpha_{0, j}^{\prime} \in \Omega_{0}^{\boldsymbol{a}^{\prime}}$ and $\alpha_{0, j}^{\prime \prime} \in \Omega_{0}^{\boldsymbol{a}^{\prime \prime}}$, where $\boldsymbol{a}^{\prime}=\boldsymbol{a}(I)$ and $\boldsymbol{a}^{\prime \prime}=\boldsymbol{a}(J)$.

Let $\Omega$ be the span of all functions $\alpha_{n, j}^{\prime \prime}$ for $n \geqslant 0$, so that $\alpha_{n, j}^{\prime \prime} \in \Omega^{a^{\prime \prime}}$. Then $\Omega$ is a vertex coalgebra in the sense of Definition 4.1 whose degree zero component is $\Omega_{0}$. The corresponding vertex algebra $V=V(\Omega)$, given by Theorem 4.1, is radical-free.

Remark 4.4. Note that the map $\Omega_{0}^{a} \rightarrow \Omega_{0} \otimes \Omega_{0}$ given by

$$
\alpha \mapsto \sum_{I \sqcup J=\{1, \ldots, l\}} \sum_{j} \alpha_{0, j}^{\prime} \otimes \alpha_{0, j}^{\prime \prime}
$$

makes $\Omega_{0}$ into a coassociative cocommutative coalgebra. The dual structure on $V_{0}$ is that of an associative commutative algebra with respect to the product $(-1)$.

Example 4.1. The main example of the coalgebra $\Omega_{0}$ that satisfies the assumptions of Theorem 4.2 is obtained in the following way. In the setup of Section 4.1, suppose that $\operatorname{deg} a_{i} \geqslant 0$. For any $\boldsymbol{a}=a_{1} \otimes \cdots \otimes a_{l} \in T(\mathcal{G})$ define $\Omega_{0}^{\boldsymbol{a}}=\left\{\alpha_{f} \mid f: V_{0} \rightarrow \mathbb{k}, f(\operatorname{Rad} V)=0\right\}$. In particular, taking $V$ to be a free vertex algebra, introduced in Section 4.3, we obtain $\Omega_{0}^{a}$ being the space of all regular $\Gamma_{\boldsymbol{a}^{-}}$-symmetric functions $\alpha \in \Phi^{l}$ such that $(\alpha)_{n}=0$ for all $n<0$, and $\operatorname{ord}_{i j} \alpha \geqslant-\operatorname{loc}\left(a_{i}, a_{j}\right)$.

Similarly, setting $\operatorname{deg} a=2$ for every $a \in \mathcal{G}$ and $\operatorname{loc}\left(a_{i}, a_{j}\right)=4$, we can take the space $\Omega_{0}^{\boldsymbol{a}}=\left(R^{l}\right)^{\Gamma_{\boldsymbol{a}}}$ of all $\Gamma_{\boldsymbol{a}}$-invariant admissible function (see Section 3.4) as another example of a family $\Omega_{0}^{\boldsymbol{a}}$, satisfying the assumptions of Theorem 4.2.

Another similar example, that we will need in Section 6.3 below, is $\Omega_{0}^{a}=\left(S^{l}\right)^{\Gamma_{a}}$, where $S^{l} \subset R^{l}$ is the space of admissible functions with only simple poles.

Proof of Theorem 4.2. Condition $(\Omega 0)$ holds because of $(\mathrm{v})$, and it is easy to see that $\Omega$ satisfies conditions $(\Omega 1),(\Omega 2)$ and $\left(\Omega 4^{\prime}\right)$. In order to show that $\Omega$ is indeed the vertex coalgebra generated by $\Omega_{0}$, we are left to check $(\Omega 3)$. 
Take a partition $\{1, \ldots, l\}=I \sqcup J$ and set

$$
\begin{aligned}
& \Delta^{\prime}=\sum_{i \in I} \partial_{z_{i}}, \quad \Delta^{\prime \prime}=\sum_{i \in J} \partial_{z_{i}}, \\
& \Delta^{* \prime}=\sum_{i \in I} z_{i}^{2} \partial_{z_{i}}+\left(2 \operatorname{deg} a_{i}\right) z_{i}, \quad \Delta^{* \prime \prime}=\sum_{i \in J} z_{i}^{2} \partial_{z_{i}}+\left(2 \operatorname{deg} a_{i}\right) z_{i} .
\end{aligned}
$$

For a function $\alpha \in \Omega_{0}^{a}$, apply $\Delta$ and $\Delta^{*}$ to the expansion (3.1), and get

$$
\begin{aligned}
& 0=\Delta \alpha=\sum_{n \geqslant 0} \sum_{j}\left(\Delta^{\prime} \alpha_{n j}^{\prime}\right) \alpha_{n j}^{\prime \prime}+\alpha_{n j}^{\prime}\left(\Delta^{\prime \prime} \alpha_{n j}^{\prime \prime}\right), \\
& 0=\Delta^{*} \alpha=\sum_{n \geqslant 0} \sum_{j}\left(\Delta^{* \prime} \alpha_{n j}^{\prime}\right) \alpha_{n j}^{\prime \prime}+\alpha_{n j}^{\prime}\left(\Delta^{* \prime \prime} \alpha_{n j}^{\prime \prime}\right) .
\end{aligned}
$$

From this we deduce that

$$
\begin{aligned}
& 0=(\Delta \alpha)_{n}=\sum_{j}\left(\Delta^{\prime} \alpha_{n j}^{\prime}\right) \alpha_{n j}^{\prime \prime}+\alpha_{n+1, j}^{\prime}\left(\Delta^{\prime \prime} \alpha_{n+1, j}^{\prime \prime}\right), \\
& 0=\left(\Delta^{*} \alpha\right)_{n}=\sum_{j}\left(\Delta^{* \prime} \alpha_{n j}^{\prime}\right) \alpha_{n j}^{\prime \prime}+\alpha_{n-1, j}^{\prime}\left(\Delta^{* \prime \prime} \alpha_{n-1, j}^{\prime \prime}\right),
\end{aligned}
$$

which implies that $\Delta^{\prime} \alpha_{n j}^{\prime} \in \operatorname{Span}\left\{\alpha_{n+1, j}^{\prime}\right\}, \Delta^{\prime \prime} \alpha_{n j}^{\prime \prime} \in \operatorname{Span}\left\{\alpha_{n-1, j}^{\prime \prime}\right\}, \Delta^{* \prime} \alpha_{n j}^{\prime} \in \operatorname{Span}\left\{\alpha_{n-1, j}^{\prime}\right\}$, and $\Delta^{* \prime \prime} \alpha_{n j}^{\prime \prime} \in \operatorname{Span}\left\{\alpha_{n+1, j}^{\prime \prime}\right\}$.

Now we show that $\operatorname{Rad}(V)=0$. First we observe that $\operatorname{Rad}(V)_{0}=0$, since the correlation functions of degree 0 on $V$ being regular implies that $D^{*} V_{1}=0$. Now assume that there is a homogeneous element $0 \neq v \in \operatorname{Rad}(V)$ of degree $n>0$ and weight $\lambda=b_{1}+\cdots+b_{l}$ for $b_{i} \in \mathcal{G}$. Then there is a functional $f: V_{n}^{\lambda} \rightarrow \mathbb{k}$ such that $f(v) \neq 0$. Let $\beta\left(z_{1}, \ldots, z_{l}\right) \in \Omega_{n}^{b}$ for $\boldsymbol{b}=b_{1} \otimes \cdots \otimes b_{l}$ be the corresponding correlation function. By the construction of $\Omega$ we can assume that $\beta$ is the coefficient of some monomial $w_{1}^{-m_{1}-1} \cdots w_{k}^{-m_{k}-1}$ in the power series expansion of a function $\alpha\left(w_{1}, \ldots, w_{k}, z_{1}, \ldots, z_{l}\right) \in \Omega_{0}^{\boldsymbol{a} \otimes \boldsymbol{b}}$ in the domain $\left|w_{1}\right|>\cdots>\left|w_{k}\right|$, where $\boldsymbol{a}=a_{1} \otimes \cdots \otimes a_{k}$. But then $a_{1}\left(m_{1}\right) \cdots a_{k}\left(m_{k}\right) v \neq 0$ in $V_{0}$, which contradicts to the fact that $v \in \operatorname{Rad}(V)$.

\subsection{The component of degree zero}

Suppose $\mathcal{G}, T(\mathcal{G})$, loc and $\Gamma_{\boldsymbol{a}}$ for $\boldsymbol{a} \in T(\mathcal{G})$ are as in Section 4.1. Here we prove the following fact:

Theorem 4.3. Assume that for any $\boldsymbol{a} \in T(\mathcal{G})$ we are given a space $\Phi^{\boldsymbol{a}} \subset \Phi^{l}$, such that the space $\Omega_{0}=\bigoplus_{a \in T(\mathcal{G})} \Omega_{0}^{a}$, defined by $\Omega_{0}^{a}=\left(\Phi^{a}\right)^{\Gamma_{a}}$, satisfies the assumptions of Theorem 4.2. Assume also that $\Phi^{\boldsymbol{a}} \Phi^{\boldsymbol{b}} \subset \Phi^{\boldsymbol{a} \otimes \boldsymbol{b}}$ for any $\boldsymbol{a}, \boldsymbol{b} \in T(\mathcal{G})$. Let $V=V(\Omega)$ be the vertex algebra constructed in Theorem 4.2. Then $V_{0}$ is isomorphic to a polynomial algebra.

Note that the spaces $\Omega_{0}$ given in Example 4.1 are all obtained in this way.

Before proving this theorem, we need to establish certain property of the algebra $V_{0}$. We know that $V_{0}$ is an associative commutative algebra, graded by weights: $V_{0}=\bigoplus_{\lambda \in \mathbb{Z}_{+}[\mathcal{G}]} V_{0}^{\lambda}$. Let $X=\bigoplus_{\lambda \neq 0} V_{0}^{\lambda}$ be the augmentation ideal in $V_{0}$. Consider the symmetrized tensor product $\operatorname{Sym}_{X}^{2} X=\left(X \otimes_{X} X\right)_{\Sigma_{2}}$. There is the canonical homomorphism $\mu: \operatorname{Sym}_{X}^{2} X \rightarrow X^{2}$ defined by $\mu(x \otimes y)=x y$.

Lemma 4.2. The map $\mu: \operatorname{Sym}_{X}^{2} X \rightarrow X^{2}$ is an isomorphism. 
Proof. Clearly, $\mu$ is surjective. To prove that it is also injective, suppose that $\sum_{i} u_{i} v_{i}=0$ in $X^{2} \subset V_{0}$ for some homogeneous $u_{i}, v_{i} \in X$. We need to show that $\sum_{i} u_{i} \otimes v_{i}=0$ in $\operatorname{Sym}_{X}^{2} X$.

The tensor product $\operatorname{Sym}_{X}^{2} X$ is graded by $\mathbb{Z}_{+}[\mathcal{G}]$. Therefore, it is enough to check that $f\left(\sum_{i} u_{i} \otimes v_{i}\right)=0$ for any homogeneous linear functional $f: \operatorname{Sym}_{X}^{2} X \rightarrow \mathbb{k}$. Assume that wt $f=\lambda=a_{1}+\cdots+a_{l} \in \mathbb{Z}_{+}[\mathcal{G}]$. For a non-trivial partition $P=\left\{P_{1}, P_{2}\right\} \in \overline{\mathcal{P}}_{2}$, set $\lambda^{\prime}=\sum_{i \in P_{1}} a_{i}$ and $\lambda^{\prime \prime}=\sum_{i \in P_{2}} a_{i}$. Then $f$ can be pulled back to a functional on $V_{0}^{\lambda^{\prime}} \otimes V_{0}^{\lambda^{\prime \prime}}$. Since both $V_{0}^{\lambda^{\prime}}$ and $V_{0}^{\lambda^{\prime \prime}}$ are finite-dimensional, we can write this functional as $\sum_{j} f_{j}^{\prime} \otimes f_{j}^{\prime \prime}$ for some $f_{j}^{\prime}: V_{0}^{\lambda^{\prime}} \rightarrow \mathbb{k}$ and $f_{j}^{\prime \prime}: V_{0}^{\lambda^{\prime \prime}} \rightarrow \mathbb{k}$.

Set $\boldsymbol{a}=a_{1} \otimes \cdots \otimes a_{l}$ and $\boldsymbol{a}^{\prime}=\boldsymbol{a}\left(P_{1}\right), \boldsymbol{a}^{\prime \prime}=\boldsymbol{a}\left(P_{2}\right)$ as in Section 4.1. Let $\alpha_{j}^{\prime} \in \Omega_{0}^{\boldsymbol{a}^{\prime}}$ and $\alpha_{j}^{\prime \prime} \in \Omega_{0}^{a^{\prime \prime}}$ be the correlation functions of $f_{j}^{\prime}$ and $f_{j}^{\prime \prime}$ respectively. Set

$$
\alpha(P)=\sum_{j} \alpha_{j}^{\prime} \alpha_{j}^{\prime \prime}
$$

Denote $\Gamma=\Gamma_{\boldsymbol{a}}$. We claim that the functions $\alpha(P) \in \Phi^{\boldsymbol{a}}$ for $P \in \overline{\mathcal{P}}_{2}$ satisfy the properties of Proposition 3.1 and also $\alpha(\sigma P)=\alpha(P)$ for any $\sigma \in \Gamma$.

Note that one of the assumptions of Theorem 4.2 was that $\left(\alpha^{\prime}\right)_{d}=\left(\alpha^{\prime \prime}\right)_{d}=0$ for $d<0$, therefore $(\alpha(Q))_{0}(P)$ is the leading term in the expansion (3.1) of a function $\alpha(Q)$. The condition (3.2) follows from the fact that $a b \otimes c d=a c \otimes b d$ in $\operatorname{Sym}_{X}^{2} X$ for every $a, b, c, d \in X$.

So by Proposition 3.1 there exists a function $\alpha \in \Phi^{a}$ such that $(\alpha)_{0}(P)=\alpha(P)$ for any partition $P \in \overline{\mathcal{P}}$. Replacing $\alpha$ by $|\Gamma|^{-1} \sum_{\sigma \in \Gamma} \sigma \alpha$ we can assume that $\alpha \in \Omega_{0}^{a}$. Then $\alpha$ is a correlation function of a linear functional $h: V_{0}^{\lambda} \rightarrow \mathbb{k}$, such that $h\left(u_{i} v_{i}\right)=f\left(u_{i} \otimes v_{i}\right)$ for any pair $u_{i}, v_{i}$. Therefore, $f\left(\sum_{i} u_{i} \otimes v_{i}\right)=h\left(\sum_{i} u_{i} v_{i}\right)=0$.

Proof of Theorem 4.3. Recall that the augmentation ideal $X=\bigoplus_{l>0} X_{l}$ of $V_{0}$ is graded, where

$$
X_{l}=\bigoplus_{\boldsymbol{a} \in T(\mathcal{G}),|\boldsymbol{a}|=l} V_{0}^{\boldsymbol{a}} .
$$

For $v \in X_{l}$ we will call $l=|v|$ the length of $v$. Choose a homogeneous basis $\mathcal{X} \subset X$ of $X$ modulo $X^{2}$. We want to show that $V_{0} \cong \mathbb{k}[\mathcal{X}]$. Note that we can extend the grading on $\mathcal{X}$ to the grading on $\mathbb{k}[\mathcal{X}]$.

Consider the canonical map $\varphi: \mathbb{k}[\mathcal{X}] \rightarrow V_{0}$, that maps every element $x \in \mathcal{X}$ into itself. We need to show that $\varphi$ is an isomorphism. It is easy to see that $\varphi$ is surjective - this follows from the fact that for fixed length $l$, we have $X_{l} \cap X^{k}=0$ for $k \gg 0$.

Let $\bar{X}=\mathcal{X} \mathbb{k}[\mathcal{X}]$ be the augmentation ideal of $\mathbb{k}[\mathcal{X}]$. Consider the map $\psi: \bar{X}^{2} \rightarrow \operatorname{Sym}_{X}^{2} X$ that maps a monomial $x_{1} \cdots x_{k}$ to $x_{1} \otimes \varphi\left(x_{2} \cdots x_{k}\right)$ for $x_{i} \in \mathcal{X}$. Note that the space $\operatorname{Sym}_{X}^{2} X$ is graded by the length.

The restriction $\varphi: \bar{X}_{i} \rightarrow X_{i}$ is an isomorphism for the minimal $i$, because then $X_{i}=\bar{X}_{i}=$ $\operatorname{Span}\{x \in \mathcal{X}|| x \mid=i\}$. Assume we have established that $\varphi: \bar{X}_{i} \rightarrow X_{i}$ is an isomorphism for $i \leqslant l-1$. Then $\psi: \bar{X}_{i}^{2} \rightarrow\left(\operatorname{Sym}_{X}^{2} X\right)_{i}$ is an isomorphism for $i \leqslant l$. Combining this with the isomorphism of Lemma 4.2, we get an isomorphism $\bar{X}_{i}^{2} \cong X_{i}^{2}$ for $i \leqslant l$. But if $p \in \bar{X}_{l}$ is such that $\varphi(p)=0$, then $p \in \bar{X}^{2}$, since $\mathcal{X} \cup\{\mathbb{1}\}$ is linearly independent modulo $X^{2}$, therefore we must have $p \equiv 0$ and $\bar{X}_{l} \cong X_{l}$.

\section{OZ vertex algebras}

\subsection{Some notations}

Assume we have a vertex algebra $V$ graded as $V=V_{0} \oplus V_{2} \oplus V_{3} \oplus \cdots$. First of all recall (see Section 2.1) that $V_{0}$ is an associative commutative algebra under the operation $a b=a(-1) b$ 
and $V$ is a vertex algebra over $V_{0}$. Indeed, $V$ is a $V_{0}$-module under the action $a v=a(-1) v$ for $a \in V_{0}$ and $v \in V$, and the identity (V4) of Definition 2.1 implies that this action commutes with the vertex algebra structure on $V$. The component $V_{2}$ is a commutative (but not associative in general) algebra with respect to the product $a b=a(1) b$, equipped with an invariant symmetric bilinear form $\langle a \mid b\rangle=a(3) b$.

Let $A \subset V_{2}$ be a subspace such that $A(3) A \subseteq \mathbb{k} \mathbb{1} \subseteq V_{0}$ and $A(1) A \subseteq A$. Set as before $T(A)=\left\{a_{1} \otimes \cdots \otimes a_{l} \in A^{\otimes l} \mid a_{1}, \ldots, a_{l} \in A\right\}$. Denote by $V^{\prime}$ the graded dual space of $V$.

For a tensor $\boldsymbol{a}=a_{1} \otimes \cdots \otimes a_{l} \in T(A)$ and a linear functional $f \in V^{\prime}$ let $\alpha=\alpha_{f}\left(z_{1}, \ldots, z_{l}\right)$ the correlation function, given by (2.9). Then $\alpha \in R^{l}$. Indeed, $\alpha$ is regular, since $D^{*} V_{1}=0$ (see Section 3.3), has $(\alpha)_{n}=0$ for $n<0$ or $n=1$ because $V_{n}=0$ for these $n$ (see Section 3.1), has poles of order at most $4 \operatorname{since} \operatorname{loc}(a, b)=4$ for any $a, b \in A$ and has $\rho_{i j}^{(k)} \in R^{l+k / 2}$ for $k=-2,-4$ by the associativity property of correlation functions. Note that $\rho_{i j}^{(-4)} \alpha$ does not depend on $z_{i}, z_{j}$, since $a_{i}(3) a_{j} \in \mathbb{k} \mathbb{1}$. This defines a map

$$
\phi: V^{\prime} \otimes A^{\otimes l} \rightarrow R^{l}
$$

such that $\phi(f, \boldsymbol{a})=\sigma \phi(f, \sigma \boldsymbol{a})$ for any $\boldsymbol{a} \in A^{\otimes l}, f \in V^{\prime}$ and $\sigma \in \Sigma_{l}$.

There is an obvious action of the symmetric groups and of $\mathrm{k}^{\times}$on $T(A)$. Set $S(A)=$ $T(A)_{\Sigma \times \mathbb{k}^{\times}}=P T(A)_{\Sigma}$. Denote by $\Omega^{\boldsymbol{a}}=\left\{\phi(f, \boldsymbol{a}) \mid f \in V^{\prime}\right\} \subset R^{l}$ the space of all correlation functions corresponding to $\boldsymbol{a}$.

Recall that for $\boldsymbol{a}=a_{1} \otimes \cdots \otimes a_{l} \in T(A)$ we have considered the space

$$
V^{\boldsymbol{a}}=\operatorname{Span}_{\mathbb{k}}\left\{a_{1}\left(m_{1}\right) \cdots a_{l}\left(m_{l}\right) \mathbb{1} \mid m_{i} \in \mathbb{Z}\right\},
$$

so that $V^{\boldsymbol{a}} \cong\left(\Omega^{\boldsymbol{a}}\right)^{\prime}$. If $\boldsymbol{a}=\boldsymbol{b}$ in $S(A)$, then $V^{\boldsymbol{a}}=V^{\boldsymbol{b}}$. Set also

$$
V^{(l)}=\operatorname{Span}_{\mathbb{k}}\left\{a_{1}\left(m_{1}\right) \cdots a_{k}\left(m_{k}\right) \mathbb{1} \mid a_{i} \in A, m_{i} \in \mathbb{Z}, k \leqslant l\right\},
$$

so that we have a filtration $\mathbb{k}_{k} \mathbb{1}=V^{(0)} \subseteq V^{(1)} \subseteq V^{(2)} \subseteq \cdots \subseteq V$. Denote $V_{d}^{(l)}=V^{(l)} \cap V_{d}$.

Let $\mathcal{G} \subset A$ be a linear basis of $A$. Then $\mathbb{Z}_{+}[\mathcal{G}]$ can be identified with a subset of $S(A)$ by $a_{1}+\cdots+a_{l}=a_{1} \otimes \cdots \otimes a_{l}$ for $a_{i} \in \mathcal{G}$. Every tensor $\boldsymbol{a} \in T(A)$ of length $l$ can be expanded as $\boldsymbol{a}=\sum_{i} k_{i} \boldsymbol{g}_{i}$ for $k_{i} \in \mathbb{k}$ and $\boldsymbol{g}_{i} \in T(\mathcal{G})$. This implies that

$$
V^{(l)}=\bigcup_{\lambda \in \mathbb{Z}_{+}[\mathcal{G}],|\lambda| \leqslant l} V^{\lambda}
$$

Next we define the maps $r_{i j}^{(1)}, r_{i j}^{(3)}: T(A) \rightarrow T(A)$ by

$$
\begin{aligned}
& r_{i j}^{(1)} a_{1} \otimes \cdots \otimes a_{l}=a_{1} \otimes \cdots \otimes a_{i-1} \otimes a_{i} a_{j} \otimes \cdots \otimes \widehat{a}_{j} \otimes \cdots a_{l}, \\
& r_{i j}^{(3)} a_{1} \otimes \cdots \otimes a_{l}=\left\langle a_{i} \mid a_{j}\right\rangle a_{1} \otimes \cdots \otimes a_{i-1} \otimes \widehat{a}_{i} \otimes \cdots \otimes \widehat{a}_{j} \otimes \cdots a_{l} .
\end{aligned}
$$

It follows from the associativity property of Section 2.2 that

$$
\rho_{i j}^{(-2)} \phi(f, \boldsymbol{a})=\phi\left(f, r_{i j}^{(1)} \boldsymbol{a}\right), \quad \rho_{i j}^{(-4)} \phi(f, \boldsymbol{a})=\phi\left(f, r_{i j}^{(3)} \boldsymbol{a}\right)
$$

for all $f \in V^{\prime}$.

Define a partial ordering on $T(A)$ by writing $\boldsymbol{b} \prec \boldsymbol{a}$ if $\boldsymbol{b}=r_{i j}^{(k)} \boldsymbol{a}$, and taking the transitive closure. 


\subsection{Virasoro element}

Now we investigate what happens when an element $\omega \in A \subset V_{2}$ is a Virasoro element of $V$ (see Section 2.1). Recall that in this case $\omega(0) a=D a$ and $\omega(1) a=(\operatorname{deg} a) a$ for every homogeneous $a \in V$, see (2.7), and therefore $\frac{1}{2} \omega$ is an idempotent in the Griess algebra $V_{2}$.

Consider a tensor $\boldsymbol{a}=a_{1} \otimes \cdots \otimes a_{l-1} \otimes \omega \in T(A)$, and set

$$
\boldsymbol{b}=r_{i l}^{(1)} \boldsymbol{a}=2 a_{1} \otimes \cdots \otimes a_{l-1}, \quad \boldsymbol{b}_{i}=r_{i l}^{(3)} \boldsymbol{a}=\left\langle\omega \mid a_{i}\right\rangle a_{1} \otimes \cdots \otimes \widehat{a}_{i} \otimes \cdots \otimes a_{l-1} .
$$

Let $f \in V_{0}^{\prime}$ be a linear functional. Denote $\alpha\left(z_{1}, \ldots, z_{l}\right)=\phi(f, \boldsymbol{a}), \beta\left(z_{1}, \ldots, z_{l-1}\right)=\phi(f, \boldsymbol{b})$ and $\beta_{i}\left(z_{1}, \ldots, \widehat{z}_{i}, \ldots, z_{l-1}\right)=\phi\left(f, \boldsymbol{b}_{i}\right)$.

Define an operator $\mathcal{E}: \Phi^{l-1} \rightarrow \Phi^{l-1}$ by

$$
\mathcal{E}=\sum_{i=1}^{l-1}-z_{i}^{-1} \partial_{z_{i}}+2 z_{i}^{-2},
$$

and let the shift operator $T: \Phi^{l-1} \rightarrow \Phi^{l}$ be given by $T\left(f\left(z_{1}, \ldots, z_{l-1}\right)\right)=f\left(z_{1}-z_{l}, \ldots, z_{l-1}-z_{l}\right)$.

Proposition 5.1. Suppose that $V$ is generated by $A \subset V_{2}$ as a vertex algebra. Then an element $\omega \in A$ is a Virasoro element of $V$ if and only if

$$
\alpha=\frac{1}{2} T \mathcal{E} \beta+\sum_{i=1}^{l-1}\left(z_{i}-z_{l}\right)^{-4} \beta_{i}
$$

for every $\boldsymbol{a}=a_{1} \otimes \cdots \otimes a_{l-1} \otimes \omega \in T(A)$ and $f \in V_{0}^{\prime}$.

Remark 5.1. If $\langle\omega \mid \omega\rangle \neq 0$, we can choose a basis $\omega \in \mathcal{G} \subset A$ so that $\omega$ is orthogonal to the rest of basic elements. Then it is enough to check (5.6) only for $a_{1} \otimes \cdots \otimes a_{l-1} \in T(\mathcal{G})$, and the second sum runs over the indices $i$ such that $a_{i}=\omega$.

Proof. If $\omega \in V_{2}$ is a Virasoro element, then by $(2.15)$ we have $[a(m), \omega(-1)]=(m+1) a(m-$ $2)+\delta_{m, 3}\langle\omega \mid a\rangle$ for any $a \in A$, which implies

$$
[Y(a, z), \omega(-1)]=\left(2 z^{-2}-z^{-1} \partial_{z}\right) Y(a, z)+\langle\omega \mid a\rangle z^{-4} \mathbb{1}
$$

Therefore,

$$
\begin{aligned}
\alpha\left(z_{1}, \ldots, z_{l-1}, 0\right)= & f\left(Y\left(a_{1}, z_{1}\right) \cdots Y\left(a_{l-1}, z_{l-1}\right) \omega(-1) \mathbb{1}\right) \\
= & \mathcal{E} f\left(Y\left(a_{1}, z_{1}\right) \cdots Y\left(a_{l-1}, z_{l-1}\right) \mathbb{1}\right) \\
& \left.+\sum_{i=1}^{l-1}\left\langle\omega \mid a_{i}\right\rangle z_{i}^{-4} f\left(Y\left(a_{1}, z_{1}\right) \cdots Y \widehat{\left(a_{i}, z_{i}\right.}\right) \cdots Y\left(a_{l-1}, z_{l-1}\right) \mathbb{1}\right) \\
= & \frac{1}{2} \mathcal{E} \beta\left(z_{1}, \ldots, z_{l-1}\right)+\sum_{i=1}^{l-1} z_{i}^{-4} \beta_{i}\left(z_{1}, \ldots, \widehat{z}_{i}, \ldots, z_{l-1}\right),
\end{aligned}
$$

and we get (5.6) since $\alpha\left(z_{1}, \ldots, z_{l}\right)=\alpha\left(z_{1}-z_{l}, \ldots, z_{l-1}-z_{l}, 0\right)$ by Proposition 3.2.

Conversely, in order to see that $\omega \in A$ is a Virasoro element, we need to show that ad $\omega(1)$ : $U(V) \rightarrow U(V)$ is the grading derivation and ad $\omega(0): U(V) \rightarrow U(V)$ coincides with $D$. Since $A$ generates $V$ as a vertex algebra, the operators $a(n)$ for $a \in A, n \in \mathbb{Z}$, generate $U(V)$ as an associative algebra, and therefore it is enough to verify commutation relations between $\omega(m)$ and $a(n)$ for $m=0,1$ and $n \in \mathbb{Z}$. Using (2.15) this amounts to checking the identities

$$
\omega(0) a=D a \quad \text { and } \quad \omega(1) a=2 a
$$


for any $a \in A$. Note that we also have $\omega(2) a=0$ since $V_{2}=0$ and $\omega(3) a=\langle\omega \mid a\rangle \mathbb{1}$ since $\omega \in A$. Using (2.4), these identities are equivalent to

$$
a(0) \omega=D a, \quad a(1) \omega=2 a, \quad \forall a \in A .
$$

Setting $a_{l-1}=a$ and $z_{l-1}=z$ we expand

$$
\alpha\left(z_{1}, \ldots, z_{l-1}, 0\right)=\sum_{n \geqslant 0} z^{-4+n} \alpha_{n}\left(z_{1}, \ldots, z_{l-2}\right),
$$

where $\alpha_{n}=f\left(Y\left(a_{1}, z_{1}\right) \cdots Y\left(a_{l-2}, z_{l-2}\right) a(3-n) \omega\right)$. It follows that $(5.7)$ is equivalent to

$$
\begin{aligned}
\alpha_{2}\left(z_{1}, \ldots, z_{l-2}\right) & =f\left(Y\left(a_{1}, z_{1}\right) \cdots Y\left(a_{l-2}, z_{l-2}\right) a(1) \omega\right) \\
& =2 f\left(Y\left(a_{1}, z_{1}\right) \cdots Y\left(a_{l-2}, z_{l-2}\right) a\right)=\beta\left(z_{1}, \ldots, z_{l-2}, 0\right) \\
\alpha_{3}\left(z_{1}, \ldots, z_{l-2}\right) & =f\left(Y\left(a_{1}, z_{1}\right) \cdots Y\left(a_{l-2}, z_{l-2}\right) a(0) \omega\right) \\
& =f\left(Y\left(a_{1}, z_{1}\right) \cdots Y\left(a_{l-2}, z_{l-2}\right) D a\right)=\left.\frac{1}{2} \frac{\partial \beta}{\partial z_{l-1}}\right|_{z_{l-1}=0}
\end{aligned}
$$

which easy follows from (5.6).

\subsection{The operator $\mathcal{E}$}

In this section we show that the operator $\mathcal{E}$ preserves the property of being admissible (see Section 3.4).

Proposition 5.2. For an admissible function $\beta \in R^{l-1}, l \geqslant 3$, set $\alpha=T \mathcal{E} \beta$.

a. For any $1 \leqslant i<l$ we have

$$
\rho_{i l}^{(-1)} \alpha=-\partial_{z_{i}} \beta, \quad \rho_{i l}^{(-2)} \alpha=2 \beta
$$

and $\rho_{i l}^{(k)} \alpha=0$ for $k<-2$.

b. For any $1 \leqslant i<j<l$ we have

$$
\rho_{i j}^{(-2)} \alpha=T \mathcal{E} \rho_{i j}^{(-2)} \beta+2\left(z_{j}-z_{l}\right)^{-4} \rho_{i j}^{(-4)} \beta, \quad \rho_{i j}^{(-4)} \alpha=T \mathcal{E} \rho_{i j}^{(-4)} \beta
$$

and $\rho_{i j}^{(k)} \alpha=0 \quad$ for $k<-4$.

c. $\alpha \in R^{l}$.

Proof. (a) Since $\beta$ and $\partial_{z_{i}} \beta$ are translation-invariant, we have

$$
T \mathcal{E} \beta=\sum_{i=1}^{l-1}\left(-\left(z_{i}-z_{l}\right)^{-1} \partial_{z_{i}}+2\left(z_{i}-z_{l}\right)^{-2}\right) \beta\left(z_{1}, \ldots, z_{l-1}\right),
$$

therefore

$$
\alpha=2\left(z_{i}-z_{l}\right)^{-2} \beta-\left(z_{i}-z_{l}\right)^{-1} \partial_{z_{i}} \beta+O\left(\left(z_{i}-z_{l}\right)^{0}\right) .
$$

(b) Assume for simplicity that $i=1$ and $j=2$. Expand

$$
\beta=\left(z_{1}-z_{2}\right)^{-4} \beta_{-4}\left(z_{3}, \ldots, z_{l-1}\right)+\left(z_{1}-z_{2}\right)^{-2} \beta_{-2}\left(z_{2}, \ldots, z_{l-1}\right)+\cdots .
$$


Using that $\left[\mathcal{E}, z_{1}-z_{2}\right]=z_{1}^{-1} z_{2}^{-1}\left(z_{1}-z_{2}\right)$, we get

$$
\mathcal{E}\left(z_{1}-z_{2}\right)^{k}=\left(z_{1}-z_{2}\right)^{k}\left(\mathcal{E}+k z_{1}^{-1} z_{2}^{-1}\right) .
$$

So we compute

$$
\begin{aligned}
\mathcal{E}\left(z_{1}-z_{2}\right)^{-2} \beta_{-2} & =\left(z_{1}-z_{2}\right)^{-2}\left(\mathcal{E}^{\prime}+2 z_{1}^{-2}-2 z_{1}^{-1} z_{2}^{-1}\right) \beta_{-2} \\
& =\left(z_{1}-z_{2}\right)^{-2} \mathcal{E}^{\prime} \beta_{-2}+O\left(\left(z_{1}-z_{2}\right)^{-1}\right), \\
\mathcal{E}\left(z_{1}-z_{2}\right)^{-4} \beta_{-4} & =\left(z_{1}-z_{2}\right)^{-4}\left(\mathcal{E}^{\prime \prime}+2 z_{1}^{-2}+2 z_{2}^{-2}-4 z_{1}^{-1} z_{2}^{-1}\right) \beta_{-4} \\
& =\left(z_{1}-z_{2}\right)^{-4} \mathcal{E}^{\prime \prime} \beta_{-4}+2\left(z_{1}-z_{2}\right)^{-2} z_{2}^{-4} \beta_{-4}+O\left(\left(z_{1}-z_{2}\right)^{-1}\right),
\end{aligned}
$$

where $\mathcal{E}^{\prime}=\sum_{i=2}^{l-1} z_{1}^{-1} \partial_{z_{1}}-2 z_{1}^{-2}, \quad \mathcal{E}^{\prime \prime}=\sum_{i=3}^{l-1} z_{1}^{-1} \partial_{z_{1}}-2 z_{1}^{-2}$.

(c) First we show that $\alpha$ is regular. Set $\Delta_{1}^{*}=\sum_{i=1}^{l-1} z_{i}^{2} \partial_{z_{i}}+4 z_{i}$ and $\Delta^{*}=\Delta_{1}^{*}+z_{l}^{2} \partial_{z_{l}}+4 z_{l}$. It is enough to check that $\Delta_{1}^{*}(\mathcal{E} \beta)\left(z_{1}, \ldots, z_{l-1}\right)=0$. Indeed, in this case set $w_{i}=z_{i}-z_{l}$ for $1 \leqslant i \leqslant l-1$, and get

$$
\begin{aligned}
\Delta^{*} T(\mathcal{E} \beta) & =\left(\left(\sum_{i=1}^{l-1} z_{i}^{2} \partial_{w_{i}}+4 z_{i}\right)-\left(\sum_{i=1}^{l-1} z_{l}^{2} \partial_{w_{i}}\right)+4 z_{l}\right) \mathcal{E} \beta\left(w_{1}, \ldots, w_{l-1}\right) \\
& =\left(\Delta_{1}^{*}+z_{l}\left(4 l+2 \sum_{i=1}^{l-1} w_{i} \partial_{w_{i}}\right)\right) \mathcal{E} \beta\left(w_{1}, \ldots, w_{l-1}\right) \\
& =(4 l+2 \operatorname{deg} \mathcal{E} \beta) z_{l} \mathcal{E} \beta\left(w_{1}, \ldots, w_{l-1}\right)=0,
\end{aligned}
$$

since $\operatorname{deg} \mathcal{E} \beta=\operatorname{deg} \beta-2=-2 l$. So we compute $\left[z^{2} \partial_{z}+4 z,-z^{-2} \partial_{z}+2 z\right]=3 \partial_{z}$, hence $\left[\Delta_{1}^{*}, \mathcal{E}\right]=$ $3 \sum_{i=1}^{l-1} \partial z_{i}$, and therefore, using Proposition 3.2 , we get $\Delta_{1}^{*} \mathcal{E} \beta=\mathcal{E} \Delta_{1}^{*} \beta+3 \sum_{i} \partial_{z_{i}} \beta=0$.

In order to finish the proof of (c) we need only to show that for every partition $\{1, \ldots, l\}=$ $I \sqcup J$ the expansion (3.1) of $\alpha$ has form

$$
\alpha=(\alpha)_{0}+\sum_{n \geqslant 2}(\alpha)_{n}
$$

so that $(\alpha)_{0}=\sum_{j} \alpha_{0, j}^{\prime} \alpha_{0, j}^{\prime \prime}$ for $\alpha_{0, j}^{\prime} \in R^{|I|}$ and $\alpha_{0, j}^{\prime \prime} \in R^{|J|}$.

We prove this statement by induction on $l$. If $l=3$, then $\beta=k\left(z_{1}-z_{2}\right)^{-4}$ for $k \in \mathbb{k}$ and then $\alpha=2 k\left(z_{1}-z_{2}\right)^{-2}\left(z_{1}-z_{3}\right)^{-2}\left(z_{2}-z_{3}\right)^{-2} \in R^{3}$ and the statements (a) and (b) are obviously true. So assume that $l \geqslant 4$.

Without loss of generality we can assume that $l \in J$. Write the expansion (3.1) for $\beta$ as $\beta=(\beta)_{0}+\sum_{n \geqslant 2}(\beta)_{n}$ where $(\beta)_{n}=\sum_{j} \beta_{n, j}^{\prime} \beta_{n, j}^{\prime \prime}$. Note that both $(\beta)_{0}$ and $\sum_{n \geqslant 2}(\beta)_{n}$ are admissible. Then

$$
\alpha=\sum_{n \geqslant 0, n \neq 1} T \mathcal{E}(\beta)_{n}=\sum_{n, j}\left(T \mathcal{E}^{\prime} \beta_{n, j}^{\prime}\right) \beta_{n, j}^{\prime \prime}+\beta_{n, j}^{\prime}\left(T \mathcal{E}^{\prime \prime} \beta_{n, j}^{\prime \prime}\right),
$$

where $\mathcal{E}^{\prime}=\sum_{i \in I}-z_{i}^{-1} \partial_{z_{i}}+2 z_{i}^{-2}$ and $\mathcal{E}^{\prime \prime}=\sum_{i \in J \backslash\{l\}}-z_{i}^{-1} \partial_{z_{i}}+2 z_{i}^{-2}$. By induction, $T \mathcal{E}^{\prime} \beta_{0, j}^{\prime} \in$ $R^{|I|+1}$ and $T \mathcal{E}^{\prime \prime} \beta_{0, j}^{\prime \prime} \in R^{|I|}$, therefore $T \mathcal{E}(\beta)_{0} \in R^{l}$.

We are left to show that $\left(T \mathcal{E}(\beta)_{n}\right)_{m}=0$ for $n \geqslant 2$ and $m \leqslant 1$. Observe that $T \mathcal{E}^{\prime} \beta_{n, j}^{\prime}$ does not have pole at $z_{l}$, therefore

$$
\left(\left(T \mathcal{E}^{\prime} \beta_{n, j}^{\prime}\right) \beta_{n, j}^{\prime \prime}\right)_{m}=0
$$

for $m<n$, and the claim follows. 


\subsection{Explicit formulae for $\Omega_{0}^{a}$ for small $a$}

Let $\boldsymbol{a}=a_{1} \otimes \cdots \otimes a_{l} \in T(A)$. It follows from (5.4) that if $f: V_{0}^{\boldsymbol{a}} \rightarrow \mathbb{k}$ is such that $f\left(V^{(l-1)}\right)=0$, then the corresponding correlation function $\phi(f, \boldsymbol{a}) \in \Omega_{0}^{\boldsymbol{a}}$ has only simple poles. The smallest such function is

$$
\prod_{1 \leqslant i<j \leqslant 5}\left(z_{i}-z_{j}\right)^{-1} \in R^{5}
$$

therefore for $l \leqslant 4$ the space of correlation functions $\Omega_{0}^{a}$ has dimension 1 . We have $\Omega_{0}^{1}=\mathbb{k}$, $\Omega_{0}^{a}=0$, and for $l=2,3,4, \Omega_{0}^{a}=\mathbb{k} \alpha$, where $\alpha$ is as follows:

$$
\begin{aligned}
l=2: & \alpha=\left\langle a_{1} \mid a_{2}\right\rangle\left(z_{1}-z_{2}\right)^{-4} \\
l=3: & \alpha=\left\langle a_{1} \mid a_{2} a_{3}\right\rangle\left(z_{1}-z_{2}\right)^{-2}\left(z_{1}-z_{3}\right)^{-2}\left(z_{2}-z_{3}\right)^{-2} \\
l=4: & \alpha=\left\langle a_{1} \mid a_{2}\right\rangle\left\langle a_{3} \mid a_{4}\right\rangle\left(z_{1}-z_{2}\right)^{-4}\left(z_{3}-z_{4}\right)^{-4} \\
& +\left\langle a_{1} \mid a_{3}\right\rangle\left\langle a_{2} \mid a_{4}\right\rangle\left(z_{1}-z_{3}\right)^{-4}\left(z_{2}-z_{4}\right)^{-4} \\
& +\left\langle a_{1} \mid a_{4}\right\rangle\left\langle a_{2} \mid a_{3}\right\rangle\left(z_{1}-z_{4}\right)^{-4}\left(z_{2}-z_{3}\right)^{-4} \\
& +\left\langle a_{1} a_{2} \mid a_{3} a_{4}\right\rangle\left(z_{1}-z_{2}\right)^{-2}\left(z_{3}-z_{4}\right)^{-2}\left(z_{1}-z_{3}\right)^{-1}\left(z_{1}-z_{4}\right)^{-1}\left(z_{2}-z_{3}\right)^{-1}\left(z_{2}-z_{4}\right)^{-1} \\
& +\left\langle a_{1} a_{3} \mid a_{2} a_{4}\right\rangle\left(z_{1}-z_{3}\right)^{-2}\left(z_{2}-z_{4}\right)^{-2}\left(z_{1}-z_{2}\right)^{-1}\left(z_{1}-z_{4}\right)^{-1}\left(z_{2}-z_{3}\right)^{-1}\left(z_{3}-z_{4}\right)^{-1} \\
& +\left\langle a_{1} a_{4} \mid a_{2} a_{3}\right\rangle\left(z_{1}-z_{4}\right)^{-2}\left(z_{2}-z_{3}\right)^{-2}\left(z_{1}-z_{2}\right)^{-1}\left(z_{1}-z_{3}\right)^{-1}\left(z_{2}-z_{4}\right)^{-1}\left(z_{3}-z_{4}\right)^{-1} .
\end{aligned}
$$

\section{The algebra $B$}

Now let $A$ be a commutative algebra with a symmetric invariant bilinear form $\langle\cdot \mid \cdot\rangle$. Denote by Aut $A$ its the group of automorphisms, i.e. the linear maps that preserve the product and the form on $A$. In this section we prove the following theorem:

Theorem 6.1. There exists a vertex algebra $B=B_{0} \oplus B_{2} \oplus B_{3} \oplus \cdots$, generated by $A \subset B_{2}$, so that

a. $a(1) b=a b$ and $a(3) b=\langle a \mid b\rangle$ for any $a, b \in A$;

b. if $\frac{1}{2} \omega \in A$ is a unit of $A$, then $\omega$ is a Virasoro element of $B$;

c. Aut $A \subset$ Aut $B$.

d. If $\operatorname{dim} A=1$ or, if $A$ has a unit $\frac{1}{2} \omega, \operatorname{dim} A / \mathbb{k} \omega=1$, then $B_{0}=\mathbb{k}$; otherwise, $B_{0}$ is isomorphic to the polynomial algebra in infinitely many variables.

Remark 6.1. In fact one can show that the vertex algebra $B$ can be obtained as $B=\widehat{B} / \operatorname{Rad} \widehat{B}$, where $\widehat{B}$ is the vertex algebra generated by the space $A$ subject to relations (a) of Theorem 6.1 and condition $\widehat{B}_{1}=0$.

Before constructing the algebra $B$ and proving Theorem 6.1, let us show how it implies the main result of this paper.

\subsection{Proof of Theorem 1.1}

Assume that the form $\langle\cdot \mid \cdot\rangle$ on $A$ is non-degenerate. Take an arbitrary algebra homomorphism $\chi: B_{0} \rightarrow \mathbb{k}$. If a finite group of automorphisms $G \subset$ Aut $A$ was specified, choose $\chi$ to be $G$ symmetric. (Here we use that char $\mathbb{k}=0$.) By Proposition 2.1, $\chi$ defines a $\mathbb{k}$-valued symmetric 
bilinear form $\langle\cdot \mid \cdot\rangle_{\chi}$ on $B$, such that $\langle a \mid b\rangle_{\chi}=\chi\left(a(-1)^{*} b\right)$. It is easy to see that the form $\langle\cdot \mid \cdot\rangle_{\chi}$ coincides with the form $\langle\cdot \mid \cdot\rangle$ on $A$. Indeed, for $a, b \in A$ we have, using that $\chi(\mathbb{1})=1$,

$$
\langle a \mid b\rangle_{\chi}=\chi\left(a(-1)^{*} b\right)=a(3) b \chi(\mathbb{1})=\langle a \mid b\rangle .
$$

Since $\chi$ is multiplicative, we have $\operatorname{Ker}_{\chi} \subset \operatorname{Rad}\langle\cdot \mid \cdot\rangle_{\chi}$. Now we set

$$
V=B / \operatorname{Rad}\langle\cdot \mid \cdot\rangle_{\chi}
$$

Since $V_{0}=\mathbb{k} \mathbb{1}$, Proposition 2.2c implies that $V$ is simple. This proves Theorem 1.1a, whereas (b) and (c) of Theorem 1.1 follow from (b) and (c) of Theorem 6.1.

\subsection{Constructing $B_{0}$}

Let us fix a linear basis $\mathcal{G}$ of $A$, such that if $A$ has a unit $\frac{1}{2} \omega$, then $\omega \in \mathcal{G}$. First we construct the spaces

$$
\mathrm{k} \mathbb{1}=B_{0}^{(0)} \subseteq B_{0}^{(1)} \subseteq \cdots \subseteq B_{0},
$$

defined by (5.2). Recall that $T(A)=\left\{a_{1} \otimes a_{2} \otimes \cdots \mid a_{i} \in A\right\} \subset \bigoplus_{l \geqslant 1} A^{\otimes l}$ and $S(A)=T(A)_{\Sigma \times \mathbb{k} \times}$. For any $\boldsymbol{a} \in T(A)$ of length $|\boldsymbol{a}| \leqslant l$ we will construct a subspace $B_{0}^{\boldsymbol{a}} \subset B_{0}^{(l)}$ and the dual space of admissible correlation functions $\left(B_{0}^{a}\right)^{*}=\Omega_{0}^{a} \subset R^{l}$, so that the following properties will hold:

B1. $B_{0}^{\boldsymbol{a}}=B_{0}^{\boldsymbol{b}}$ if $\boldsymbol{a}=\boldsymbol{b}$ in $S(A)$.

B2. $B_{0}^{\boldsymbol{b}} \subseteq B_{0}^{\boldsymbol{a}}$ whenever $\boldsymbol{b} \prec \boldsymbol{a}$.

B3. There is a map

$$
\phi:\left(B_{0}^{a}\right)^{*} \otimes A^{\otimes l} \rightarrow \Omega_{0}^{a},
$$

for $\boldsymbol{a} \in T(A),|\boldsymbol{a}|=l$, satisfying (5.4) and $\phi(f, \boldsymbol{a})=\sigma \phi(f, \sigma \boldsymbol{a})$ for any permutation $\sigma \in \Sigma_{l}$.

B4. If $\frac{1}{2} \omega \in A$ is a unit, then for any $\boldsymbol{b} \in T(A),|\boldsymbol{b}|=l-1, \boldsymbol{a}=\boldsymbol{b} \otimes \omega, \quad \boldsymbol{b}_{i}=r_{i j}^{(3)} \boldsymbol{a}$ and $f: B^{(l)} \rightarrow \mathbb{k}$

$$
\phi(f, \boldsymbol{a})=T \mathcal{E} \phi(f, \boldsymbol{b})+\sum_{i=1}^{l-1}\left(z_{i}-z_{l}\right)^{-4} \phi\left(f, \boldsymbol{b}_{i}\right) .
$$

B5. $B_{0}^{(l)} / B_{0}^{(l-1)}=\bigoplus_{\lambda \in \mathbb{Z}_{+}[\mathcal{G} \backslash\{\omega\}]} B_{0}^{\lambda} / B_{0}^{(l-1)}$.

In addition we want $\Omega_{0}^{a}$ to satisfy the conditions (i)-(vi) of Theorem 4.2.

The map $\phi$ in (B3) is going to be the same as in (5.1). As in Section 4.1, the condition (B3) implies that $\Omega^{a} \subset R^{\Gamma a}$. The condition (B4) is the same as in Proposition 5.1. The property (B1) justifies the notation $B_{0}^{\lambda}=B_{0}^{\boldsymbol{a}}$ used in (B5), whenever $\lambda=g_{1}+\cdots+g_{l} \in \mathbb{Z}_{+}[\mathcal{G}]$ and $\boldsymbol{a}=$ $g_{1} \otimes \cdots \otimes g_{l} \in T(\mathcal{G})$. The property (B5) is a special case of (5.3).

We are constructing $B_{0}^{(l)}$ by induction on $l$, starting from $B_{0}^{(0)}=\mathbb{k} \mathbb{1}$ and $\Omega_{0}^{1}=\mathbb{k}$. Assume that $B_{0}^{(m)}, \Omega_{0}^{\boldsymbol{a}}$ and $\phi:\left(B_{0}^{(m)}\right)^{*} \otimes A^{\otimes m} \rightarrow R^{m}$ are already constructed for $m=|\boldsymbol{a}| \leqslant l-1$. 


\section{Constructing $\Omega_{0}^{g}$}

Take a basic tensor $\boldsymbol{g}=g_{1} \otimes \cdots \otimes g_{l} \in T(\mathcal{G})$. We define the space $\Omega_{0}^{\boldsymbol{g}} \subset R^{l}$ in the following way: If $g_{i} \neq \omega$ for all $1 \leqslant i \leqslant l$, then set

$$
\Omega_{0}^{\boldsymbol{g}}=\left\{\begin{array}{l|c}
\alpha \in\left(R^{l}\right)^{\Gamma \boldsymbol{g}} & \begin{array}{c}
\exists f: B_{0}^{(l-1)} \rightarrow \mathbb{k} \text { s.t. } \rho_{i j}^{(-k-1)} \alpha=\phi\left(f, r_{i j}^{(k)} \boldsymbol{g}\right) \\
\forall 1 \leqslant i<j \leqslant l, k=1 \text { or } 3
\end{array}
\end{array}\right\} .
$$

It is not clear a priori why $\Omega_{0}^{g} \neq 0$. This is a part of the statement of Proposition 6.1 below. Note also that by fixing some $0 \neq \alpha \in \Omega_{0}^{g}$, the space $\Omega_{0}^{g}$ can be described as the space of functions that differ from $\alpha$ by an admissible $\Gamma_{\boldsymbol{g}}$-symmetric function with only simple poles.

If $g_{l}=\omega$, then set $\boldsymbol{b}=r_{i l}^{(1)} \boldsymbol{g}, \boldsymbol{b}_{i}=r_{i l}^{(3)} \boldsymbol{g} \in T(\mathcal{G})$ as in (5.5), and then define $\Omega_{0}^{\boldsymbol{g}}$ to be set of all functions $\alpha \in R^{l}$ given by (5.6), where $\beta=\phi^{l-1}(f, \boldsymbol{b}), \beta_{i}=\phi^{l-2}\left(f, \boldsymbol{b}_{i}\right)$ for all $f: B_{0}^{(l-1)} \rightarrow \mathrm{k}$. Note that $\alpha \in R^{l}$ due to Proposition 5.2c.

Finally, if $g_{i}=\omega$ for some $1 \leqslant i \leqslant l-1$, then set

$$
\Omega_{0}^{\boldsymbol{g}}=(i l) \Omega_{0}^{(i l) \boldsymbol{g}},
$$

for the transposition $(i l) \in \Sigma_{l}$.

It is immediately clear that for any permutation $\sigma \in \Sigma_{l}$ we have

$$
\Omega_{0}^{\boldsymbol{g}}=\sigma \Omega_{0}^{\sigma \boldsymbol{g}} .
$$

The following proposition gives another crucial property of the functions $\Omega_{0}^{g}$.

Proposition 6.1. For any linear functional $f: B_{0}^{(l-1)} \rightarrow \mathbb{k}$ and any tensor $\boldsymbol{g}=g_{1} \otimes \cdots \otimes g_{l} \in$ $T(\mathcal{G})$ there is a function $\alpha \in \Omega_{0}^{g}$ such that $\rho_{i j}^{(-k-1)} \alpha=\phi\left(f, r_{i j}^{(k)} \boldsymbol{g}\right)$.

Proof. In the case when $\boldsymbol{g}$ does not contain $\omega$, Proposition 3.4 guarantees that there exists an admissible function $\alpha \in R^{l}$ such that $\rho_{i j}^{(-k-1)} \alpha=\phi\left(f, r_{i j}^{(k)} \boldsymbol{g}\right)$, since the functions $\alpha_{i j}^{(k)}=$ $\phi\left(f, r_{i j}^{(-k-1)} \boldsymbol{g}\right)$ obviously satisfy the condition (3.6). Now take $\left|\Gamma_{\boldsymbol{g}}\right|^{-1} \sum_{\sigma \in \Gamma_{\boldsymbol{g}}} \sigma \alpha \in \Omega_{0}^{\boldsymbol{g}}$.

Now suppose that $\boldsymbol{g}$ contains $\omega$. Using (6.2), we can assume without loss of generality, that $\boldsymbol{g}=a_{1} \otimes \cdots \otimes a_{l-1} \otimes \omega$. Let $\boldsymbol{b}=r_{i l}^{(1)} \boldsymbol{g}$ and $\boldsymbol{b}_{s}=r_{s l}^{(3)} \boldsymbol{g}$ for $1 \leqslant s<l$ as in (5.5), and set $\beta=\phi(f, \boldsymbol{b}), \quad \beta_{s}=\phi\left(f, \boldsymbol{b}_{s}\right)$. Then $\alpha=\phi(f, \boldsymbol{g}) \in \Omega_{0}^{\boldsymbol{g}}$ is defined by (5.6). By Proposition 5.2a, we get $\rho_{i l}^{(-2)} \alpha=\beta$ and $\rho_{i l}^{(-4)} \alpha=\beta_{i}$ for all $1 \leqslant i<l$.

Now consider the case when $1 \leqslant i<j<l$. Applying $\rho_{i j}^{(-4)}$ to (5.6) and using Proposition 5.2b, we get

$$
\rho_{i j}^{(-4)} \alpha=T \mathcal{E} \rho_{i j}^{(-4)} \beta+\sum_{s \neq i, j}\left(z_{s}-z_{l}\right)^{-4} \rho_{i j}^{(-4)} \beta_{s}=\phi\left(f, r_{i j}^{(3)} \boldsymbol{g}\right) .
$$

To do the same with $\rho_{i j}^{(-2)}$ we notice that

$$
r_{j l}^{(3)} r_{i j}^{(1)} \boldsymbol{g}=r_{i j}^{(3)} \boldsymbol{b} .
$$

Indeed, assuming that $i, j=1,2$ to simplify notations, we get

$$
\begin{aligned}
r_{2 l}^{(3)} r_{12}^{(1)} \boldsymbol{g} & =r_{2 l}^{(3)}\left(a_{1} a_{2}\right) \otimes a_{3} \otimes \cdots \otimes a_{l-1} \otimes \omega \\
& =\left\langle\omega \mid a_{1} a_{2}\right\rangle a_{3} \otimes \cdots \otimes a_{l-1}=2\left\langle a_{1} \mid a_{2}\right\rangle a_{3} \otimes \cdots \otimes a_{l-1}=r_{12}^{(3)} \boldsymbol{b} .
\end{aligned}
$$

Now we apply $\rho_{i j}^{(-2)}$ to (5.6) and compute, using Proposition 5.2b,

$$
\rho_{i j}^{(-2)} \alpha=T \mathcal{E} \rho_{i j}^{(-2)} \beta+\left(z_{j}-z_{l}\right)^{-4} \rho_{i j}^{(-4)} \beta+\sum_{s \neq i, j}\left(z_{s}-z_{l}\right)^{-4} \rho_{i j}^{(-2)} \beta_{s}=\phi\left(f, r_{i j}^{(1)} \boldsymbol{g}\right) .
$$




\section{Constructing $B_{0}^{(l)}$}

For $\boldsymbol{g} \in T(\mathcal{G})$ set $B_{0}^{\boldsymbol{g}}=\left(\Omega_{0}^{\boldsymbol{g}}\right)^{*}$. For a permutation $\sigma \in \Sigma_{l}$ we identify $B_{0}^{\boldsymbol{g}}$ with $B_{0}^{\sigma \boldsymbol{g}}$ by setting $(b, \alpha)=\left(b, \sigma^{-1} \alpha\right)$ for $b \in B_{0}^{\sigma \boldsymbol{g}}$ and $\alpha \in \Omega_{0}^{g}$, since $\sigma^{-1} \alpha \in \Omega^{\sigma \boldsymbol{g}}$ by (6.2). Thus for a weight $\lambda=g_{1}+\cdots+g_{l} \in \mathbb{Z}_{+}[\mathcal{G}]$ we can denote $B_{0}^{\lambda}=B_{0}^{g}$, where $\boldsymbol{g}=g_{1} \otimes \cdots \otimes g_{l} \in T(\mathcal{G})$.

By the construction, for any function $\alpha \in \Omega_{0}^{g}$ there is a linear functional $f: B_{0}^{(l-1)} \rightarrow \mathbb{k}$ such that $\rho_{i j}^{(m)} \alpha=\phi\left(f, r_{i j}^{(-m-1)} \boldsymbol{g}\right)$ for all $1 \leqslant i<j \leqslant l$ and $m=-2,-4$. Take a tensor $\boldsymbol{g} \succ \boldsymbol{a} \in T(A)$. There is a map $\rho_{\boldsymbol{g a}}: \Omega_{0}^{\boldsymbol{g}} \rightarrow \Omega_{0}^{\boldsymbol{a}}$, which is an iteration of the maps $\rho_{i j}^{(m)}$, so that

$$
\rho_{\boldsymbol{g a}} \alpha=\phi(f, \boldsymbol{a}) .
$$

We note that the restriction of $f$ on $B_{0}^{\boldsymbol{a}}$ is uniquely defined by $\alpha$, so $\rho_{\boldsymbol{g a}}$ is well defined by (6.3).

By Proposition 6.1, the map $\rho_{\boldsymbol{g a}}: \Omega_{0}^{\boldsymbol{g}} \rightarrow \Omega_{0}^{\boldsymbol{a}}$ is surjective, therefore we have an embedding $\rho_{\boldsymbol{g a}}^{*}: B_{0}^{\boldsymbol{a}} \hookrightarrow B_{0}^{\boldsymbol{g}}$. Set

$$
B_{0}^{(l)}=\left(B_{0}^{(l-1)} \oplus \bigoplus_{\substack{\lambda \in \mathbb{Z}_{+}[\mathcal{G}] \\|\lambda|=l}} B_{0}^{\lambda}\right) / \operatorname{Span}\left\{a-\rho_{\boldsymbol{g} \boldsymbol{a}}^{*} a \mid a \in B_{0}^{\boldsymbol{a}}, T(\mathcal{G}) \ni \boldsymbol{g} \succ \boldsymbol{a} \in T(A)\right\} .
$$

In other words, we identify the space $B_{0}^{\boldsymbol{a}} \subset B_{0}^{(l-1)}$ with the subspace $\rho_{\boldsymbol{g a}}^{*}\left(B_{0}^{\boldsymbol{a}}\right) \subset B_{0}^{\boldsymbol{g}}$ for $\boldsymbol{g} \succ \boldsymbol{a} \in$ $T(A)$. So we have $B_{0}^{(l-1)} \subset B_{0}^{(l)}$ and $B_{0}^{\lambda} \subset B_{0}^{(l)}$ for any $\lambda \in \mathbb{Z}_{+}[\mathcal{G}]$ of length $l$.

For $\boldsymbol{g} \in T(\mathcal{G}),|\boldsymbol{g}|=l$, we define the map $\phi(\cdot, \boldsymbol{g}):\left(B_{0}^{(l)}\right)^{*} \rightarrow \Omega_{0}^{\boldsymbol{g}} \subset R^{l}$ in the following way. Since $\left(B_{0}^{\boldsymbol{g}}\right)^{*} \cong \Omega_{0}^{\boldsymbol{g}}$, the restriction of a functional $f: B_{0}^{(l)} \rightarrow \mathbb{k}$ to $B_{0}^{\boldsymbol{g}}$ can be identified with a function in $\Omega_{0}^{g}$, and we set $\phi(f, \boldsymbol{g})=\left.f\right|_{B_{0}^{g}}$. Then we extend $\phi$ by linearity to the map $\phi:\left(B_{0}^{(l)}\right)^{*} \otimes A^{\otimes l} \rightarrow R^{l}$.

\section{Verification of (B1)-(B5)}

The properties (B1) and (B3) are clear. For a tensor $\boldsymbol{a} \in T(A),|\boldsymbol{a}|=l$, consider the restriction $\phi(\cdot, \boldsymbol{a}):\left(B_{0}^{(l)}\right)^{*} \rightarrow R^{l}$. Denote its image by $\Omega_{0}^{\boldsymbol{a}} \subset R^{l}$. The the dual map $\phi(\cdot, \boldsymbol{a})^{*}: B_{0}^{\boldsymbol{a}} \rightarrow B_{0}^{(l)}$ is an embedding of the dual space $B_{0}^{\boldsymbol{a}}=\left(\Omega_{0}^{\boldsymbol{a}}\right)^{*}$ into $B_{0}^{(l)}$. This establishes (B2).

If $\boldsymbol{a} \in T(A)$ ends by $\omega$, and $\boldsymbol{b}$ and $\boldsymbol{b}_{i}$ are defined as in (5.5), then any function $\alpha \in \Omega^{\boldsymbol{a}}$ is uniquely defined by its coefficients $\rho_{i l}^{(-2)} \alpha=\beta \in \Omega^{\boldsymbol{b}}$ and $\rho_{i l}^{(-4)} \alpha=\beta_{i} \in \Omega^{\boldsymbol{b}_{i}}$ by the formula (5.6). This implies (B4). Note also that in this case

$$
B^{\boldsymbol{a}} \subseteq B^{\boldsymbol{b}}+B^{\boldsymbol{b}_{1}}+\cdots+B^{\boldsymbol{b}_{l-1}} \subset B^{(l-1)} .
$$

The condition (B5) follows from (6.4).

\section{Verification of conditions (i) - (vi) of Theorem 4.2}

The only conditions of Theorem 4.2 that require verification are (v) and (vi). If we assume that the form $\langle\cdot \mid \cdot\rangle$ on $A$ is non-degenerate, then for any $a \in \mathcal{G}$ there is $b \in \mathcal{G}$ such that $\langle a \mid b\rangle \neq 0$, and then $\Omega_{0}^{a \otimes b}=\mathbb{k}\left(z_{1}-z_{2}\right)^{-4} \neq 0$, which proves (v). Another argument, that does not use non-degeneracy of $\langle\cdot \mid \cdot\rangle$, can be found in the proof of Theorem 6.1d in Section 6.3 below.

To prove (vi), consider a partition $\{1, \ldots, l\}=I \sqcup J$ and set $\boldsymbol{a}^{\prime}=\boldsymbol{a}(I), \boldsymbol{a}^{\prime \prime}=\boldsymbol{a}(J) \in T(\mathcal{G})$ (in the notations of Section 4.1). Since $\alpha \in R^{l}$, we have $(\alpha)_{n}=\sum_{j} \alpha_{-n, j}^{\prime} \alpha_{n, j}^{\prime \prime}=0$ for $n<0$ or $n=1$ by Definition 3.2, and $\alpha_{0, j}^{\prime}, \alpha_{0, j}^{\prime \prime}$ are admissible by Proposition 3.3c. Denote $\boldsymbol{b}=r_{i j}^{(k)} \boldsymbol{a}$ and 
$\boldsymbol{b}^{\prime}=\boldsymbol{b}(I)$. To show that $\alpha_{0, j}^{\prime} \in \Omega_{0}^{\boldsymbol{a}^{\prime}}$ we need to show that $\rho_{i j}^{(-k-1)} \alpha_{0, j}^{\prime} \in \Omega_{0}^{\boldsymbol{b}^{\prime}}$, for any $i, j \in I$ and $k=1$ or 3 . But this follows from (vi) applied to $\rho_{i j}^{(-k-1)} \alpha$, since we obviously have

$$
\left(\rho_{i j}^{(-k-1)} \alpha\right)_{0}=\sum_{s}\left(\rho_{i j}^{(-k-1)} \alpha_{0, j}^{\prime}\right)\left(\alpha_{0, j}^{\prime \prime}\right) .
$$

Similarly, we check that $\alpha_{0, j}^{\prime \prime} \in \Omega_{0}^{\boldsymbol{a}^{\prime \prime}}$.

\subsection{Proof of Theorem 6.1}

We apply Theorem 4.2 to the space of functions $\Omega_{0}$ constructed in Section 6.2, and obtain a vertex algebra

$$
\widehat{B}=\bigoplus_{n \geqslant 0, n \neq 1} \widehat{B}_{n}, \quad \widehat{B}_{n}=\bigoplus_{\lambda \in \mathbb{Z}_{+}[\mathcal{G}]} \widehat{B}_{n}^{\lambda} .
$$

Note that we have $\widehat{B}_{1}=0$ due to the fact that $\Omega_{0}$ consists of admissible functions which do not have components of degree 1 , see Definition 3.2.

From the construction of $B_{0}$ we see that $\widehat{B}_{0}^{\lambda}=B_{0}^{\lambda}$ for any $\lambda \in \mathbb{Z}_{+}[\mathcal{G}]$. Recall that $\widehat{B}_{0}$ is the associative commutative algebra, dual to the coalgebra $\Omega_{0}$ with respect to the coproduct (4.7). But then $B_{0}$ is also an associative commutative algebra, since $B_{0}^{\lambda} \subset B_{0}$, and we have a surjective algebra homomorphism $\pi: \widehat{B}_{0} \rightarrow B_{0}$. Its kernel Ker $\pi$ is an ideal in $\widehat{B}_{0}$, which by Proposition 2.2c can be extended to an ideal $\overline{\operatorname{Ker} \pi} \subset \widehat{B}$. So we finally set

$$
B=\widehat{B} / \overline{\operatorname{Ker} \pi} \text {. }
$$

The condition (a) holds by the construction: indeed, given $\boldsymbol{a}=a_{1} \otimes a_{2} \otimes a_{3} \otimes \cdots \in T(A)$, and a correlation function $\alpha \in \Omega^{a}$, the coefficient $\rho_{12}^{(-2)} \alpha$ is the correlation function corresponding to $\left(a_{1} a_{2}\right) \otimes a_{3} \otimes \cdots$, but by the associativity condition of Section 2.2 it must be the correlation function for $\left(a_{1}(1) a_{2}\right) \otimes a_{3} \otimes \cdots$, which implies that $a_{1}(1) a_{2}=a_{1} a_{2}$. The equality $a_{1}(3) a_{2}=$ $\left\langle a_{1} \mid a_{2}\right\rangle$ is established in the same way.

If $\frac{1}{2} \omega \in A$ is a unit, then any correlation function $\alpha \in \Omega_{0}^{\boldsymbol{a} \otimes \omega}$ is given by the formula (5.6), therefore, $\omega$ is a Virasoro element by Proposition 5.1, thus proving (b). Note also that the construction of $B$ was canonical, which establishes (c).

\section{Proof of Theorem 6.1d}

We need to introduce another vertex algebra. Let $F=V(\Omega(F))$ be the vertex algebra obtained by the construction of Theorem 4.2 from the space of functions

$$
\Omega(F)_{0}=\bigoplus_{\boldsymbol{g} \in T(\mathcal{G} \backslash\{\omega\})} \Omega(F)_{0}^{\boldsymbol{g}}
$$

where $\Omega(F)_{0}^{\boldsymbol{g}}=\left(S^{l}\right)^{\Gamma_{\boldsymbol{g}}}$ is the space of $\Gamma_{\boldsymbol{g}^{-}}$-symmetric admissible functions with only simple poles (see Example 4.1). By Theorem 4.3 the algebra $F_{0}$ is polynomial. Note that the algebra $F$ is what Theorem 6.1 would yield if instead of $A$ one would take the space Span $\{\mathcal{G} \backslash\{\omega\}\}$ with zero product and form.

On the other hand, the vertex algebra $B$ inherits a filtration (6.1) from $B_{0}$. Consider the associated graded algebra gr $B=\bigoplus_{l \geqslant 1} B^{(l)} / B^{(l-1)}$. This is indeed a vertex algebra, since all vertex algebra identities (see Definition 2.1) are homogeneous. By (B5) we have

$$
\operatorname{gr} B=\bigoplus_{\lambda \in \mathbb{Z}_{+}[\mathcal{G} \backslash\{\omega\}]}(\operatorname{gr} B)^{\lambda} .
$$


From the construction in Section 6.2 it follows that the coalgebra of correlation functions $\Omega(\operatorname{gr} B)_{0}$ is the same as $\Omega(F)_{0}$, therefore $\Omega(\operatorname{gr} B)=\Omega(F)$ and hence by Proposition 4.1 there is a vertex algebra isomorphism $\eta:(\operatorname{gr} B) \rightarrow F$, which yields algebra isomorphism $\eta_{0}:(\operatorname{gr} B)_{0}=$ $\operatorname{gr}\left(B_{0}\right) \rightarrow F_{0}$. But since a polynomial algebra cannot have non-trivial deformations, we must have $B_{0} \cong F_{0}$ as associative commutative algebras.

We are left with estimating the size of $F_{0}$. Take some $\boldsymbol{g}=a_{1} \otimes \cdots \otimes a_{l} \in T(\mathcal{G} \backslash\{\omega\})$ and let $\Gamma=\Gamma_{\boldsymbol{g}}$. A function $\alpha \in \Omega_{0}^{\boldsymbol{g}}=\left(S^{l}\right)^{\Gamma}$ can have a pole at $z_{i}-z_{j}$ only if $a_{i} \neq a_{j}$. Therefore, since $\operatorname{deg} \alpha=-2 l$, if $\mathcal{G}$ has no more than one element other than $\omega$, then $\left(S^{l}\right)^{\Gamma}=0$ for $l>0$, and hence $F_{0}=\mathrm{k}$.

Now assume that $\mathcal{G}$ has at least two elements other then $\omega$, say $a$ and $b$. Denote by $S_{0}^{l} \subset S^{l}$ the space of indecomposable admissible functions with only simple poles. Then the span of the generators of degree $\boldsymbol{g}$ of $F_{0}$ is isomorphic to $\left(S_{0}^{l}\right)^{\Gamma}$. We claim that for $l$ large enough there is $\boldsymbol{g} \in T(\mathcal{G} \backslash\{\omega\}),|\boldsymbol{g}|=l$, such that the $\left(S_{0}^{l}\right)^{\Gamma} \neq 0$. This would imply that $F_{0}$ is a polynomial algebra in infinitely many variables.

Indeed, there are infinitely many bipartite 4-regular connected graphs that remain connected after a removal of any two edges. Let $G$ be such a graph with vertices $u_{1}, \ldots, u_{k}, v_{k+1}, \ldots, v_{l}$, so that an edge can only connect some $u_{i}$ with some $v_{j}$. The incidence matrix of this graph is an $l \times l$ symmetric regular matrix $\mathrm{S}=\left\{\mathrm{s}_{\mathrm{ij}}\right\}_{\mathrm{i}, \mathrm{j}=1}$ (see Example 3.1), defined so that $s_{i j}=-1$ whenever $G$ has an edge connecting $u_{i}$ and $v_{j}$ for some $1 \leqslant i \leqslant k<j \leqslant l$, the rest of the entries being 0 . Then

$$
0 \neq|\Gamma|^{-1} \sum_{\sigma \in \Gamma} \sigma \pi(\mathrm{S}) \in\left(\mathrm{S}_{0}^{1}\right)^{\Gamma}=\Omega_{0}^{g}
$$

where $\pi(\mathrm{S})$ is as in (3.4) and $\boldsymbol{g}=a \otimes \cdots \otimes a \otimes b \otimes \cdots \otimes b$.

\section{References}

[1] Borcherds R.E., Vertex algebras, Kac-Moody algebras, and the Monster, Proc. Nat. Acad. Sci. U.S.A. 83 (1986), 3068-3071.

[2] Borcherds R.E., Monstrous moonshine and monstrous Lie superalgebras, Invent. Math. 109 (1992), $405-444$.

[3] Dong C., Representations of the moonshine module vertex operator algebra, in Mathematical Aspects of Conformal and Topological Field Theories and Quantum Groups (South Hadley, MA, 1992), Contemp. Math. 175 (1994), 27-36.

[4] Dong C., Li H., Mason G., Montague P.S., The radical of a vertex operator algebra, in The Monster and Lie algebras (Columbus, OH, 1996), Ohio State Univ. Math. Res. Inst. Publ., Vol. 7, de Gruyter, Berlin, 1998, 17-25, q-alg/9608022.

[5] Dong C., Lin Z., Mason G., On vertex operator algebras as $\mathrm{sl}_{2}$-modules, in Groups, Difference Sets, and the Monster (Columbus, OH, 1993), Ohio State Univ. Math. Res. Inst. Publ., Vol. 4, de Gruyter, Berlin, 1996, 349-362.

[6] Dong C., Mason G., Zhu Y., Discrete series of the Virasoro algebra and the moonshine module, in Algebraic Groups and Their Generalizations: Quantum and Infinite-Dimensional Methods (University Park, PA, 1991), Proc. Sympos. Pure Math., Vol. 56, Part 2, American Mathematical Society, Providence, RI, 1994, 295-316.

[7] Frenkel I.B., Huang Y.-Z., Lepowsky J., On axiomatic approaches to vertex operator algebras and modules, Mem. Amer. Math. Soc. 104 (1993), no. 494.

[8] Frenkel I.B., Lepowsky J., Meurman A., A natural representation of the Fischer-Griess Monster with the modular function $J$ as character, Proc. Nat. Acad. Sci. U.S.A. 81 (1984), 3256-3260.

[9] Frenkel I.B., Lepowsky J., Meurman A., Vertex operator algebras and the Monster, Pure and Applied Mathematics, Vol. 134, Academic Press, Boston, MA, 1988.

[10] Frenkel I.B., Zhu Y., Vertex operator algebras associated to repersentations of affine and Virasoro algebras, Duke Math. J. 66 (1992), 123-168. 
[11] Griess R.L., The friendly giant, Invent. Math. 69 (1982), 1-102.

[12] Griess R.L., GNAVOA. I. Studies in groups, nonassociative algebras and vertex operator algebras, in Vertex Operator Algebras in Mathematics and Physics (Toronto, ON, 2000), Fields Inst. Commun., Vol. 39, American Mathematical Society, Providence, RI, 2003, 71-88.

[13] Hubbard K., The notion of vertex operator coalgebra and a geometric interpretation, Comm. Algebra 34 (2006), 1541-1589, math.QA/0405461.

[14] Kac V.G., Vertex algebras for beginners, 2nd ed., University Lecture Series, Vol. 10, American Mathematical Society, Providence, RI, 1998.

[15] Lam C.H., Construction of vertex operator algebras from commutative associative algebras, Comm. Algebra 24 (1996), 4339-4360.

[16] Lam C.H., On VOA associated with special Jordan algebras, Comm. Algebra 27 (1999), 1665-1681.

[17] Lepowsky J., Li H., Introduction to vertex operator algebras and their representations, Progress in Mathematics, Vol. 227, Birkhäuser Boston, Inc., Boston, MA, 2004.

[18] Li H., Symmetric invariant bilinear forms on vertex operator algebras, J. Pure Appl. Algebra 96 (1994), 279-297.

[19] Li H., Local systems of vertex operators, vertex superalgebras and modules, J. Pure Appl. Algebra 109 (1996), 143-195, hep-th/9406185.

[20] Li H., An analogue of the Hom functor and a generalized nuclear democracy theorem, Duke Math. J. 93 (1998), 73-114, q-alg/9706012.

[21] Primc M., Vertex algebras generated by Lie algebras, J. Pure Appl. Algebra 135 (1999), 253-293, math.QA/9901095.

[22] Roitman M., On free conformal and vertex algebras, J. Algebra 217 (1999), 496-527, math.QA/9809050.

[23] Roitman M., Combinatorics of free vertex algebras, J. Algebra 255 (2002), 297-323, math.QA/0103173.

[24] Roitman M., Invariant bilinear forms on a vertex algebra, J. Pure Appl. Algebra 194 (2004), 329-345, math.QA/0210432.

[25] Tits J., On Griess' "friendly giant", Invent. Math. 78 (1984), 491-199.

[26] Wang W., Rationality of Virasoro vertex operator algebras, Internat. Math. Res. Notices 1993 (1993), no. 7, 197-211.

[27] Xu X., Introduction to vertex operator superalgebras and their modules, Mathematics and Its Applications, Vol. 456, Kluwer Academic Publishers, Dordrecht, 1998. 